\title{
Boundedness below for fermion model theories. I
}

\author{
David Brydges* \\ Department of Mathematics, University of Michigan, Ann Arbor, Michigan 48104 \\ (Received 30 October 1974) \\ The spatially cut-off Hamiltonians for the models $(\bar{\psi} \bar{\psi} \phi)_{2}$ and $\left(\bar{\psi} \bar{\psi} \phi^{\mathrm{N}}+\phi^{2 M}\right)_{2}$ with $M>N$ are \\ bounded below uniformly in a momentum cutoff, by using the semi-Euclidean formulation.
}

\section{INTRODUCTION}

Two interactions are considered: the generalized Yukawa $\left(G Y_{2}\right)$ and the Yukawa $\left(Y_{2}\right)$. These are $\left(\lambda \psi \psi \phi^{N}\right.$ $\left.+\phi^{2 M}\right)_{1+1}$ and $(\lambda \bar{\psi} \psi \phi)_{1+1}$ respectively. The corresponding spatially cut-off Hamiltonians are shown to be bounded below provided $M>N$. For $Y_{2}$, this problem was first solved by Glimm in Ref. 1. $G Y_{2}$ was bounded below by Federbush in Ref. 2. The methods used in this paper constitute a considerable improvement over those in Ref. 2. In particular they can be extended to prove that the lower bound is linear in the volume. This will be given in another paper. Also $Y_{2}$ and $G Y_{2}$ can be treated in a uniform manner. This being so, the proof is given for $Y_{2}$, and details for $G Y_{2}$ are given only in the one place where the proofs diverge appreciably.

The lower bound is obtained by estimating, uniformly in a momentum cutoff and the expectation state,

$$
E_{0}=-\lim _{T \rightarrow \infty} \frac{1}{T} \ln \left\langle e^{-T H}\right\rangle
$$

where $H$ is given by (1.3)。For simplicity, $E_{0}$ is first estimated for the Fock vacuum. The details for establishing a bound uniform in the expectation state are provided in an appendix. $E_{0}$ is bounded below, uniformly in the expectation and momentum cutoff, provided

$$
\left\langle e^{-T H}\right\rangle \leqslant C_{1} C_{2}^{T},
$$

where $C_{2}$ is independent of the state and the cutoff.

In Sec. $2\left\langle e^{-T H}\right\rangle$ is expanded by a partly renormalized type of perturbation expansion similar to those used by Glimm and Jaffe in Refs. 3, 4. The expansion is generated by applying two identities referred to as $P$ (perturbation) and $\mathrm{C}$ (contraction). As in Ref. 4, the $P$ identity is applied in unit intervals in the time axis so that the eventual bound for $\left\langle e^{-T H}\right\rangle$ will have the form of a product of bounds over unit intervals making up $[0, T]$ as required by $(\mathbf{1}, 2)$. The expansion achieves two objectives: First the divergent quantities are exhibited and cancelled, and secondly the kernels of the remaining nondivergent quantities are rather well behaved. To increase this good behavior, one further operation is performed after the expansion is complete: The uncontracted fermion legs are given an effective momentum cutoff by moving them a short distance across neighboring exponents. This is so arranged that no further contractions occur.

In Sec. 3, by methods outlined in Ref. 5, all fermion operators are removed by an estimate that has been called "defermiation." The total Fock space is regarded as being fibered over Nelson space, and operators are estimated by taking the norm over fermion Fock space at each point in $Q$ space [Nelson space is $\left.L^{2}(Q)\right]$. The result is an expression which involves only commuting boson operators, and this can be estimated in a conventional manner. The antisymmetry of fermion wavefunctions enters into this "defermiation" in an essential way. Some of the operators are bounded pointwise in $Q$ space because of the one particle per mode property. The boundedness of the exponential operators depends on the anticommutation relations. This estimate is postponed to Sec. 7 .

In Sec. 4, the convergence of the expansion and thence (1.2) is proved with the help of an estimate on boson expectations, whose proof is postponed until Secs. 5 and 6 . The proof of convergence involves estimating sums over fermion graphs. These estimates closely follow procedures used by Dimock and Glimm in Ref. 6.

Section 5 contains an estimate on boson expectations. The methods are very similar to those in Ref. 6. The only modifications made are necessary to handle the more singular kernels resulting from the expansion, as compared with the kernels assumed in Ref. 4 . Section 6 is devoted to the proof of some estimates on kernels which are used in Secs. 4 and 5. Finally, in Sec. 7, the estimate on exponential operators, referred to in Sec. 3 , is proved. This estimate substitutes for the Wick ordering bound used in $P(\phi)_{2}$. A simple form of Glimm's dressing transformation, in which only the fermions are dressed, is used to bound from below the pair creation and annihilation part of the interaction (cf. Ref。2). In a similar way, a corresponding bound for $G Y_{2}$ can be proved, with the aid of the $\phi^{2 M}$ term in the $G Y_{2}$ interaction. Dressing only the fermions has the merit of giving a pointwise bound on $Q$ space. To complete the proof, a bound on the scattering part of the interaction is required. At this point $Y_{2}$ and $G Y_{2}$ seem to be different. In particular it is here that $M>N$ is needed, whereas $Y_{2}$ can be considered as a special case of $M=N$. This is in fact the only significant difference between the proofs for $Y_{2}$ and $G Y_{2}$. Details have been given for both. The Hamiltonian $H$ is given by

$$
\begin{aligned}
H= & H_{0 B}+H_{0 F}+\lambda \int: \bar{\psi}_{K}(x) \psi_{K}(x): \phi(x) g(x) d x \\
& -\frac{1}{2} \delta m_{K}^{2} \int g^{2}(x): \phi^{2}(x): d x-E_{K},
\end{aligned}
$$

where $H_{0 B}$ and $H_{0 F}$ are the free boson and fermion Hamiltonians:

$$
\begin{aligned}
& H_{0 B}=\int \mu(k) a^{*}(k) a(k) d k, \\
& H_{0 F}=\int \omega(p)\left[b^{*}(p) b(p)+b^{\prime *}(p) b^{\prime}(p)\right] d p .
\end{aligned}
$$

The subscript $K$ represents a sharp momentum cutoff. $g(x)$ is a nonnegative spatial cutoff satisfying 


$$
\begin{aligned}
g \in L^{p} & \forall p: 1 \leqslant p \leqslant \infty, \\
\exists \epsilon>0: \int|\hat{g}(k)|^{2}\left(1+|k|^{\epsilon}\right) d k<\infty, & \\
& \int|\hat{g}(k)|^{4 / 3}\left(1+|k|^{\epsilon}\right) d k<\infty
\end{aligned}
$$

These assumptions are quite mild: $g$ can be a characteristic function, for example. The counterterms are given by perturbation theory:

$$
\begin{aligned}
\delta m_{K}^{2}= & -\left(\frac{\lambda^{2}}{\pi}\right) \int_{|p| \leqslant K} \frac{\omega^{2}+p^{2}-M^{2}}{\omega^{2}} \frac{1}{2 \omega} d p \\
E_{K}= & -\left(\frac{\lambda}{4 \pi}\right)^{2} \int_{\substack{\left|p_{1}\right| \leqslant K \\
\left|p_{2}\right| \leqslant K}} \frac{\omega_{1} \omega_{2}-p_{1} p_{2}-M^{2}}{\omega_{1} \omega_{2}} \frac{1}{\omega_{1}+\omega_{2}+\mu(k)} \frac{1}{\mu(k)} \\
& \times\left|\hat{g}\left(p_{1}+p_{2}+k\right)\right|^{2} d p_{1} d p_{2} d k
\end{aligned}
$$

Let

$$
\begin{aligned}
V= & \lambda \int: \bar{\psi}_{K}(x) \psi_{K}(x): \phi(x) g(x) d x-\frac{1}{2} \delta m_{K}^{2} \\
& \times \int g^{2}(x): \phi^{2}(x): d x-E_{K} .
\end{aligned}
$$

The interaction is broken up in the following way:

$$
\begin{aligned}
\lambda \int: & \bar{\psi}_{K}(x) \psi_{K}(x): \phi(x) \xi(x) d x \\
= & \int_{\substack{\left|p_{1}\right| \leqslant K \\
\left|p_{2}\right| \leqslant K}}\left[b^{*}\left(p_{1}\right) b^{* *}\left(p_{2}\right)+b\left(-p_{1}\right) b^{\prime}\left(-p_{2}\right)\right] W_{p}\left(p_{1}, p_{2}, k\right) \\
& \times \phi(k) d p_{1} d p_{2} d k+\int_{\substack{\left|p_{1}\right| \leqslant K \\
\left|p_{2}\right| \leqslant K}}\left[b^{*}\left(p_{1}\right) b\left(-p_{2}\right)+b^{\prime *}\left(p_{1}\right) b^{\prime}\left(-p_{2}\right)\right. \\
& \times W_{s}\left(p_{1}, p_{2}, k\right) \phi(k) d p_{1} d p_{2} d k
\end{aligned}
$$

where

$$
\begin{aligned}
& \phi(k)=\frac{1}{\mu \frac{1}{2}(k)}\left[a^{*}(k)+a(-k)\right], \\
& W_{p}\left(p_{1}, p_{2}, k\right)=-\frac{\lambda}{(4 \pi)^{1 / 2}} \hat{g}\left(p_{1}+p_{2}+k\right)\left(\frac{\omega_{1} \omega_{2}-p_{1} p_{2}-M^{2}}{\omega_{1} \omega_{2}}\right)^{1 / 2} \\
& \times \operatorname{sgn}\left(p_{1}-p_{2}\right), \\
& W_{s}\left(p_{1}, p_{2}, k\right)=-\frac{\lambda}{(4 \pi)^{1 / 2}} \hat{g}\left(p_{1}+p_{2}+k\right)\left(\frac{\omega_{1} \omega_{2}+p_{1} p_{2}+M^{2}}{\omega_{1} \omega_{2}}\right)^{1 / 2} .
\end{aligned}
$$

The cutoffs in momentum that are used in the proof will all be selected from a sequence $\left(K_{n}\right)$ where $n$ is a positive integer. Cutoffs apply only to fermion momenta until Sec. 7, in which bosons are cut off:

$$
K_{n}=n^{\alpha} \text {. }
$$

$\alpha$ will be chosen large. The term "lower momentum cutoff" is used to indicate a momentum cutoff of the form

$J\left(p_{1}, p_{2}\right)=$ characteristic function of the set $\{$ either

$$
\left.\left|p_{1}\right| \geqslant L \text { or }\left|p_{2}\right| \geqslant L\right\} \text { for some } L \text {. }
$$

\section{THE EXPANSION}

Firstly, $\left\langle e^{-T H}\right\rangle$ is rewritten in Nelson space $N$. In Sec. 7 it is shown that a quadratic boson monomial, $c(\phi)$, depending on $K$ and an $\epsilon>0$, can be chosen so that

$$
H_{0 F}+V+c(\phi) \geqslant-O\left(K^{\epsilon}\right), \quad H_{0 B}-c(\phi) \geqslant-O(1) .
$$

By the Trotter product formula,

$$
\begin{aligned}
\left\langle e^{-T H}\right\rangle= & \lim _{N \rightarrow \infty}\left\langle\left(\exp \left\{-T / N\left[H_{0 B}-c(\phi)\right]\right\}\right.\right. \\
& \left.\left.\times \exp \left\{-T / N\left[H_{0 F}+V+c(\phi)\right]\right\}\right)^{M}\right\rangle .
\end{aligned}
$$

Define $H_{0 F}(s), V(s)$, and $c(\phi, s)$ by substituting in $H_{0 F}$, $V$, and $c(\phi)$

$$
\phi(k) \rightarrow \phi(k, s), \quad b^{\#}(p) \rightarrow b^{\#}(p, s),
$$

where $\phi(k, s)$ is the partial Fourier transform of a sharp time Euclidean boson field on Nelson space. $b^{\#}$ denotes $b^{*}, b, b^{* *}$, or $b^{\prime}$ and the time dependence in $b^{\#}(p, s)$ is dummy, i. e. , $b^{\#}(p, s)=b^{\#}(p)$. It will be used to define a time ordering.

By the Feynman-Kac-Nelson formula, as given in Ref. 7,

$$
\exp \left\{-t\left[H_{0 B}-c(\phi)\right]\right\}=E_{0} U(t) \exp \left[+\int_{0}^{t} c(\phi, \tau) d \tau\right] E_{0}
$$

[where $E_{0}$ projects onto the time zero slice of Nelson space, and $U(t)$ is the unitary operator induced by translation in time by $t]$, and the Markov property:

$$
\begin{aligned}
\left\langle e^{-T H}\right\rangle= & \lim _{N \rightarrow \infty} T\left\langle\left(\prod _ { j = 1 } ^ { N } \operatorname { e x p } \left\{-T / N\left[H_{0 F}(j T / N)+V(j T / N)\right.\right.\right.\right. \\
& \left.\left.+c(\phi, j T / N)] \exp \left[+\int_{0}^{T} c(\phi, \tau) d \tau\right]\right)\right\rangle_{N^{*}}
\end{aligned}
$$

$T$ is a time ordering operator applying to the noncommuting fermion operators. The subscript $N$ means that the expectation for the boson fields is taken in Nelson space with respect to the Nelson space vacuum. (2.5) is rewritten in the following symbolic manner:

$$
\left\langle e^{-T^{H} H}\right\rangle=T\left\langle\exp \left\{-\int_{0}^{T}\left[H_{0 F}(T)+V(\tau)\right] d \tau\right\}\right\rangle_{N} .
$$

In order to obtain an estimate with the correct dependence on $T,(2.6)$ is rewritten as

$$
\left\langle e^{-T H}\right\rangle=T\left\langle\Gamma_{I} \exp \left\{-\int_{I}\left[H_{0 F}(\tau)+V(\tau)\right] d \tau\right\}\right\rangle_{N},
$$

where $\int_{i}$ denotes integration over the time interval $[I, I+1]$ and the product runs over integers $I$ such thr: $[0, T]=\cup_{I}[I, I+1]$.

\section{Define}

$$
A(\tau)=H_{0 F}(\tau)+V(\tau), \quad A_{n}(\tau)=H_{0 F}(\tau)+V_{n}(\tau),
$$

where $V_{n}(\tau)$ is given by replacing $K$ by $K_{n}$ in (1.6). The two identities $(\mathrm{P})$ and $(\mathrm{C})$ which generate the expansion are now given. The Duhamel formula

$$
\begin{aligned}
\exp (-A)= & \exp \left(-A_{n}\right)-\int_{0}^{1} d s \exp (-s A)\left(A-A_{n}\right) \\
& \times \exp \left[-(1-s) A_{n}\right]
\end{aligned}
$$

can be applied to factors in finite time-ordered Trotter approximants and a strong limit taken to show that

$$
\begin{aligned}
T E_{\sigma_{2}} & \exp \left[-\int_{\sigma_{1}}^{\sigma_{2}} A(\tau) d \tau\right] E_{\sigma_{1}} \\
= & T E_{\sigma_{2}} \exp \left[-\int_{\sigma_{1}}^{\sigma_{2}} A_{n}(\tau) d \tau\right] E_{\sigma_{1}} \\
& -T E_{\sigma_{2}} \int_{\sigma_{1}}^{\sigma_{2}} d s\left[A(s)-A_{n}(s)\right] \\
& \times \exp \left[-\int_{\sigma_{1}}^{s} A_{n}(\tau) d \tau-\int_{s}^{\sigma_{2}} A(\tau) d \tau\right] E_{\sigma_{1}}
\end{aligned}
$$

(it is hoped that $T$, the time-ordering operator will not be confused with the time $T$ ), where $E_{\sigma_{2}}, E_{\sigma_{1}}$ project onto time slices at $\sigma_{1}$ and $\sigma_{2}$ in Nelson space and $\sigma_{1} \leqslant \sigma_{2}$. This formula remains valid if there are other operators present at times in the interval $\left[\sigma_{1}, \sigma_{2}\right]$.

By integrating the derivative of $\exp \{-(1-s)[A+\omega(p)]\}$ 
$\times b(p) \exp (-s A)$, where $b(p)$ is to be smeared by an $L^{2}$ function, it can be shown that

$$
\begin{aligned}
b(p) \exp (-A)= & \exp \{-[A+\omega(p)]\} b(p) \\
& +\int_{0}^{1} d s \exp \{-(1-s)[A+\omega(p)]\} \\
& \times[V, b(p)] \exp (-s A) .
\end{aligned}
$$

Since iterated applications of (P) will result in stepwise time dependent momentum cutoffs, $(2,10)$ is extended by allowing such cutoffs. Let

$$
A_{K}(s)=H_{0 F}(s)+V_{K}(s),
$$

where $K=K(s)$ is a stepwise time dependent momentum cutoff and $V_{K}(s)$ is defined by replacing $K$ by $K(s)$ in the definition of $V(s)$. Then it follows that for $\sigma_{2} \geqslant \sigma \geqslant \sigma_{1}$

$$
\begin{aligned}
T E_{\sigma_{2}} b(p, \sigma) \exp \left[-\int_{\sigma_{1}}^{\sigma_{2}} A K(\tau) d \tau\right] E_{\sigma_{1}} \\
=T E_{\sigma_{2}} \exp \left[-\omega(p)\left(\sigma-\sigma_{1}\right)\right] b\left(p, \sigma_{1}\right) \exp \left[-\int_{\sigma_{1}}^{\sigma_{2}} A_{K}(\tau) d \tau\right] E_{\sigma_{1}} \\
\quad+T E_{\sigma_{2}} \int_{\sigma_{1}}^{\sigma} d s \exp [-\omega(p)(\sigma-s)]\left[V_{K}(s), b(p, s)\right] \\
\quad \times \exp \left[-\int_{\sigma_{1}}^{\sigma_{2}} A K(\tau) d \tau\right] E_{\sigma_{1}} .
\end{aligned}
$$

A monomial $R=R\left(b^{\#}\left(s_{1}\right), b^{\#}\left(s_{2}\right), \ldots, b^{\#}\left(s_{n}\right)\right)$ can be included so that

$$
\begin{aligned}
T E_{\sigma_{2}} b(p, \sigma) R \exp \left[-\int_{\sigma_{1}}^{\sigma_{2}} A K(\tau) d \tau\right] E_{\sigma_{1}} \\
=T E_{\sigma_{2}} \exp \left[-\omega(p)\left(\sigma-\sigma_{1}\right)\right] b\left(p, \sigma_{1}\right) R \\
\quad \times \exp \left[-\int_{\sigma_{1}}^{\sigma_{2}} A K(\tau) d \tau\right] E_{\sigma_{1}} \\
\left.\quad+T E_{\sigma_{2}} R \int_{\sigma_{1}}^{\sigma} d s \exp [-\omega(p) \sigma-s)\right]\left[V_{K}(s), b(p, s)\right] \\
\quad \times \exp \left[-\int_{\sigma_{1}}^{\sigma_{2}} A_{K}(\tau) d \tau\right] E_{\sigma_{1}}+T \sum_{s_{i} \leqslant \sigma} E_{\sigma_{2}} \\
\quad \times R\left(\ldots, \exp \left[-\omega(p)\left(\sigma-s_{i}\right)\right]\left[b(p), b^{\#}\left(s_{i}\right)\right], \ldots\right) \\
\quad \times \exp \left[-\int_{\sigma_{1}}^{\sigma_{2}} A K(\tau) d \tau\right] E_{\sigma_{1}} \cdot
\end{aligned}
$$

There is a similar formula for $b^{\prime}$, and the adjoints are used to move $b^{*}, b^{\prime *}$ to the left.

The expansion for $\left\langle e^{-T H}\right\rangle$ is obtained as follows: An interval $I$ is selected and (P) is applied to the corresponding factor $\exp \left(-\int_{I} A d \tau\right)$ so as to interpolate between $A$ and $A_{0}$, i. e. , choose $n=0$ in (P). The interpolating term has a new " $P$ " vertex. (C) is now used to move the corresponding $b^{\# \prime} s$ over to the vacuum where they annihilate. In the course of this, new " $\mathrm{C}$ " vertices are formed; these are not further contracted. After renormalization, the description of which is postponed for the moment, (P) is applied again in the same interval to interpolate between $A$ and $A_{1}$. (C) is then applied as before, followed by renormalization, and so on. If (P) has been applied in a given interval $n$ times, then the $(n+1)$ th application in $I$ is used to interpolate between $A$ and $A_{n}$. Eventually, the expansion will terminate for $I$, because, if $n$ is large enough, $K_{n} \geqslant K$. Then a new interval is selected.

It is possible for a $\mathrm{P}$ vertex to contract twice to a $\mathrm{C}$ vertex. The corresponding factor is represented by a subgraph. See Fig. 1. The lines represent fermion contractions. $s_{\nu+1}$ and $s_{\nu}$ are the times of the vertices which are to be integrated over $s_{\nu+1}$ is to be integrated over
$[0, T]$. This will give a result that diverges as $K \rightarrow \infty$. Split the range of integration by

$$
\int_{0}^{T} d s_{\nu+1}=\int_{I} d s_{\nu+1}+\int_{I^{c}} d s_{\nu+1}
$$

The complement is with respect to $[0, T] . I$ is the interval containing the $\mathrm{P}$ vertex. The integration over $I^{c}$ is not divergent. Observe from (C) that the exponent does not depend on $s_{\nu+1}$. Furthermore, the counterterms associated with $\nu$ occur in a term with the same exponent as that in the term containing the factor $R_{\nu}$, represented by Fig. 1. Hence both terms may be combined to give a new one with a factor $(2.12)$, given below, assigned to $\nu$. For later use note that, when $\nu$ is renormalized, the cutoff in the exponent is constant in the interval $\left(s_{\nu}, I+1\right)$; also $R_{\nu}$ vanishes unless $s_{\nu+1}$ $\in\left(s_{\nu}, I+1\right)$, because otherwise the momentum cutoffs on $\nu$ and $\nu+1$ are disjoint:

$$
\left\{\int_{I} R_{\nu} d s_{\nu+1}+\frac{1}{2} \delta m_{\nu}^{2} \int g^{2}(x): \phi^{2}\left(x, s_{\nu}\right): d x+E_{\nu}\right\} .
$$

If the $\mathrm{P}$ vertex was introduced during the $n$th application of $(\mathrm{P})$ in $I$, then

$$
\delta m_{\nu}^{2}=\delta m_{K}^{2}-\delta m_{K_{n-1}}^{2}, \quad E_{\nu}=E_{K}-E_{K_{n-1}} .
$$

The expression in the curly brackets will be referred to as a "cancelled renormalization subgraph." The $C$ vertex will be referred to as having been "integrated out." Introduce the Euclidean momentum $\bar{k}=\left(k^{0}, k^{1}\right)$ and write (2.12) as

$$
\int R_{\nu}\left(\bar{k}_{1}, \bar{k}_{2}\right): \phi\left(\bar{k}_{1}\right) \phi\left(-\bar{k}_{2}\right): d \bar{k}_{1} d \bar{k}_{2}+\delta E_{\nu},
$$

where

$$
\begin{aligned}
R_{\nu}\left(\bar{k}_{1},\right. & \left.\bar{k}_{2}\right) \\
= & \int_{s_{\nu}}^{I+1} d s_{\nu+1} \int_{\substack{\left|p_{1}\right| \leqslant K \\
\left|p_{2}\right| \leqslant K}} W_{p}\left(p_{1}, p_{2}, k_{1}^{1}\right) \bar{W}_{p}\left(p_{1}, p_{2}, k_{2}^{1}\right) \rho_{n}\left(p_{1}, p_{2}\right) \\
& \quad \times \exp \left[-\left(\omega_{1}+\omega_{2}-i k_{1}^{0}\right)\left(s_{\nu+1}-s_{\nu}\right)\right] \exp \left[i\left(k_{1}^{0}-k_{2}^{0}\right) s_{\nu}\right] d p_{1} d p_{2} \\
& +\left(\frac{1}{2} \delta m_{\nu}^{2} / 4 \pi\right) \hat{g}^{*} \hat{g}\left(k_{1}^{1}-k_{2}^{1}\right) \exp \left[i\left(k_{1}^{0}-k_{2}^{0}\right) s_{\nu}\right], \\
\delta E_{\nu}= & \int_{s_{\nu}}^{I+1} d s_{\nu+1} \int\left|W_{p}\left(p_{1}, p_{2}, k\right)\right|^{2} \\
& \times \exp \left\{-\left[\omega_{1}+\omega_{2}+\mu(k)\right]\left(s_{\nu+1}-s_{\nu}\right)\right\} \\
& \times \rho_{n}\left(p_{1}, p_{2}\right) \mu^{-1}(k) d p_{1} d p_{2}+E_{\nu},
\end{aligned}
$$

where $\rho\left(p_{1}, p_{2}\right)=$ characteristic function of the set: either $\left|p_{1}\right| \geqslant K_{n}$ or $\left|p_{2}\right| \geqslant K_{n}$. For a given term in the expansion, let

$\rho(I)=$ largest fermion cutoff in the exponent in $I$.

Suppose, for this term, there are $n_{\mathrm{p}}(I) \mathrm{P}$ vertices in $I$; then by the way in which the expansion is defined and (1. 9) it follows that

$$
\rho(I)=n_{\mathrm{P}}(I)^{\alpha} \text {. }
$$

When the expansion is complete, one last operation, referred to as "smoothing the uncontracted legs," is performed. Each uncontracted fermion annihilation operator in $l$ with momentum above $\rho(I)$ is moved to the

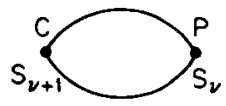

FIG. 1. A divergent subgraph. 
right, or backwards in time, halfway toward the next vertex, excluding those $\mathrm{C}$ vertices which have been integrated out because they are part of a cancelled renormalization subgraph [see (2.12)]. Similarly, uncontracted creation operators with momentum above $\rho(I)$ move to the left. Suppose such an operator associated with vertex $\nu$ is moved halfway toward vertex $\nu^{\prime}$; then the corresponding momentum acquires an effective momentum cut-off given by

$$
\exp \left[-\bar{\omega}(p)\left|s_{\nu}-s_{\nu^{\prime}}\right| / 2\right]
$$

where

$$
\begin{array}{rlrl}
\bar{\omega}(p) & =0 & & \text { if }|p| \leqslant \rho(I) \\
& =\omega(p) & \text { if }|p|>\rho(I)
\end{array}
$$

and $s_{\nu}, s_{\nu^{2}}$, are the times of the vertices $\nu$ and $\nu^{\prime}$. No contractions can occur in the course of smoothing the uncontracted legs, because the momenta of the operators that are moved are too high to contract with the exponent, and they are not moved far enough to contract with any vertex. The expansion is written in the form

$$
\left\langle e^{-T H}\right\rangle=\sum_{g} \int d s_{g} T\left\langle K_{g} \prod_{I} \exp \left(-\int_{I} B\left(\tau, s_{g}\right) d \tau\right)\right\rangle_{N},
$$

where $g$ is a label that uniquely specifies the possible vertices and their contractions. $g$ comprises:

(1) a function $I \rightarrow n(I)$ specifying the number of vertices in $l$.

(2) for each $I$, a function from $\{1,2,3, \ldots, n(I)\}$ into $\{\mathrm{P}, \mathrm{C}\}$ 。 This labels the vertices and specifies whether they are $\mathrm{P}$ or $\mathrm{C}$ vertices.

(3) a graph $F$ on $\sum_{I} n(I)$ labelled vertices with two lines leaving each vertex. Each $C$ vertex may have at most one open line, that is, a line which does not connect to another vertex. $P$ vertices have no open lines. Open lines are labelled "low momentum" or "high momentum" to specify whether they have been moved in smoothing the uncontracted legs.

An open line pointing right represents an annihilation operator; an open line pointing left represents a creation operator. Lines which are not open represent contractions resulting from using $(\mathrm{C})$.

To describe $K_{g}$, introduce the following notation:

$$
V_{\nu}\left(p_{1}, p_{2}, k\right) \text { represents either } W_{p} \text { or } W_{s}
$$

[see (1.8)]; $J_{\nu}\left(p_{1}, p_{2}\right)$ is a cutoff on the fermion momenta associated with the vertex $\nu$. In general $J_{\nu}$ will depend on $g$ and the times of the vertices. For $\mathrm{P}$ vertices $J_{\nu}$ contains a low momentum cutoff depending on $g$ and $\nu[$ see $(\mathrm{P})]$. Let

$$
\left.s_{g}=\left(s_{\nu}\right)_{\nu} \in \text { (vertices }\right\} \text {, }
$$

where $\nu$ runs over the vertices that have not been integrated out [see (2.12)]. Let $l$ denote a line in $F$ and let $t_{l}$ be the time difference associated with this line. For $l$ connecting vertices $\nu$ and $\nu^{\prime}$

$$
t_{\boldsymbol{I}}=\left|s_{\nu^{\prime}}-s_{\nu}\right| \text {. }
$$

If $l$ is an open line, then $t_{l}$ is the quantity $\left|s_{\nu}-s_{\nu^{\prime}}\right|$ given in $(2.18)$.

With each vertex $\nu$ which is not part of a cancelled renormalization subgraph (note that $\nu$ can be a vertex in a renormalization subgraph with $P$ and $C$ vertices in different intervals) associate the kernel

$$
\int Q_{\nu}\left(p_{\nu 1}, p_{\nu 2}, k\right) \phi\left(k, s_{\nu}\right) d k,
$$

where, if both lines $l_{1}$ and $l_{2}$ leaving $\nu$ are not open,

$$
\begin{aligned}
Q_{\nu}\left(p_{\nu 1}, p_{\nu 2}, k\right)= & V_{\nu}\left(p_{\nu 1}, p_{\nu 2}, k\right) J_{\nu}\left(p_{\nu 1}, p_{\nu 2}\right) \\
& \times \exp \left[-\omega\left(p_{\nu 1}\right) t_{t_{1}} / 2\right] \exp \left[-\omega\left(p_{\nu 2}\right) t_{t_{2}} / 2\right] .
\end{aligned}
$$

If one line, say $l_{1}$, is open, then

$$
\begin{aligned}
Q_{\nu}\left(p_{\nu 1}, p_{\nu 2}, k\right)= & V_{\nu}\left(p_{\nu 1}, p_{\nu 2}, k\right) J_{\nu}\left(p_{\nu 1}, p_{\nu 2}\right) \\
& \times \exp \left[-\bar{\omega}\left(p_{\nu 1}\right) t_{l_{1}} / 2\right] \exp \left[-\omega\left(p_{\nu 2}\right) t_{l_{2}} / 2\right]
\end{aligned}
$$

If $\nu$ and $\nu^{\prime}$ belong to a cancelled renormalization subgraph, and $\nu$ is the $\mathrm{P}$ vertex, then associate with $\left(\nu, \nu^{\prime}\right)$ the kernel

$$
\int R_{\nu}\left(\bar{k}_{1}, \bar{k}_{2}\right): \phi\left(\bar{k}_{1}\right) \phi\left(-\bar{k}_{2}\right): d \bar{k}_{1}, d \bar{k}_{2}+\delta E_{\nu},
$$

which is given by (2.15) and (2.26). $\tilde{K}_{g}$, the boson valued kernel of $K_{g}$, is formed by contracting the kernels (2.23a) according to the lines in the fermion graph $F$ which $g$ specifies. To contract two legs with momenta $p_{\nu 1}$, and $p_{\nu^{\prime} 1}$, say, a factor $\delta\left(p_{\nu 1}+p_{\nu^{\prime} 1}\right)$ is inserted, and the arguments are integrated over.

The quantity $B\left(\tau, s_{g}\right)$ in $(2.20)$ is defined as follows. For a given interval $I$, suppose that the $\mathrm{P}$ vertices are times $s_{1} \cdots s_{n}$ with $I=s_{0} \leqslant s_{1} \leqslant s_{2} \leqslant \cdots \leqslant s_{n} \leqslant s_{n+1}=I+1$; then for $\tau \in I$ let

$B\left(\tau, s_{g}\right)=A_{i}(\tau)$ for $s_{i} \leqslant \tau<s_{i+1}$, where $i=1,2, \ldots, n$.

The range of integration in (2.20) is such that each $C$ vertex in the interval $I$ is to be integrated over $I$. With the same definitions as in the paragraph above, the $P$ vertices in $I$ are to be integrated over the time ordered region

$$
I=s_{0} \leqslant s_{1} \leqslant \cdots \leqslant s_{n} \leqslant s_{n+1}=I+1 .
$$

\section{DEFERMIATION}

In (2.20), the operator $K_{g}$ has the form of a kernel $\widetilde{K}_{g}$, which is a function on the uncontracted fermion momenta and $Q$ space, smeared against a product of $b^{\# \prime}$ s at different times. Consider the special case wherein

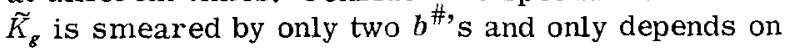
fermion modes in a finite-dimensional subspace, $S$, of the one particle fermion space. Furthermore, suppose that the operator $B\left(\tau, s_{g}\right)$ in (2.20) likewise depends only on modes in $S$. Then the fermion Fock space factors, and one need only consider the Fock space on $S$, which is a finite-dimensional Fock space. In this case, operators can be regarded as finite matrix-valued functions on $Q$ space:

$T\left\langle K_{g} \prod_{I} \exp \left(-\int_{I} B\left(\tau, s_{g}\right) d \tau\right)\right\rangle_{N}$ 


$$
\begin{aligned}
& =T\left\langle K_{g} \exp \left(-\int_{0}{ }^{T} B\left(\tau, s_{g}\right) d \tau\right)\right\rangle_{N} \\
& \leqslant\left\langle\| T\left(K_{g} \exp \left(-\int_{0}{ }^{T} B\left(\tau, s_{g}\right) d \tau\right) \|_{F}\right\rangle_{N}\right.
\end{aligned}
$$

where \|\|$_{F}$ denotes the norm over fermion Fock space at a single point in $Q$ space. It follows from the definition of the time ordered exponential that as an inequality almost everywhere on $Q$ space

$$
\begin{aligned}
\| T & {\left[K_{g} \exp \left(-\int_{0}^{T} B\left(\tau, s_{g}\right) d \tau\right)\right]_{F} } \\
& \leqslant \lim _{N \rightarrow \infty}\left\|T\left(K_{g} \prod_{i=1}^{N} \exp \left[-\frac{T}{N} B\left(\frac{i T}{N}, s_{g}\right)\right]\right)\right\|_{F} .
\end{aligned}
$$

To estimate the right-hand side, write

$$
K_{g}=\sum_{\#} \sum_{i j}\left(\tilde{K}_{g}\right)_{i j} b_{i}^{\#} b_{j}^{\#} \text { and }\left\|b_{i}^{\#}\right\|=1 \text {. }
$$

The sum over \# is over possible ways of assigning the $b^{\# \text { 's }} \mathrm{s}$ to the open lines specified by $g . i$ and $j$ refer to two orthonormal bases for $S$, and $\left(\tilde{K}_{g}\right)_{i j}$ is the matrix of $\tilde{K}_{g}$ with respect to these bases. Then (3.2) implies

$$
\begin{aligned}
& \left\|T\left[K_{g} \exp \left(-\int_{0}^{T} B\left(\tau, s_{g}\right) d \tau\right)\right]\right\|_{F} \\
& \left|\leqslant \lim _{N \rightarrow \infty} \sum_{i, j, \#}\right|\left(\tilde{K}_{g}\right)_{i j} \mid \prod_{i=1}^{N}\left\|\exp \left[-\frac{T}{N} B\left(\frac{i T}{N}, s_{g}\right)\right]\right\|_{F} .
\end{aligned}
$$

Suppose that as an operator estimate

$$
B\left(\tau, s_{g}\right) \geqslant-d\left(\phi, \tau, s_{g}\right),
$$

where $d\left(\phi, \tau, s_{g}\right)$ is a real-valued measurable function on $Q$ space. Then from (3.3):

$$
\begin{aligned}
& \left\|T\left(K_{g} \exp \left[-\int_{0}^{T} B\left(\tau, s_{g}\right) d \tau\right]\right)\right\|_{F} \\
& \quad \leqslant \sum_{i, \tilde{j}_{,}}\left|\left(\tilde{K}_{g}\right)_{i j}\right| \exp \left[\int_{0}^{T} d\left(\phi, \tau, s_{g}\right) d \tau\right] .
\end{aligned}
$$

This holds for any choice of bases in S. Taking the infimum over bases implies

$$
\begin{aligned}
& \left\|T\left(K_{g} \exp \left[-\int_{0}^{T} B\left(\tau, s_{g}\right) d \tau\right]\right)\right\|_{F} \\
& \quad \leqslant \sum_{\#} \operatorname{ir}\left|\tilde{K}_{g}\right| \exp \left[+\int_{0}^{T} d\left(\phi, \tau, s_{g}\right) d \tau\right],
\end{aligned}
$$

where $\left|\widetilde{K}_{g}\right|=\left(\tilde{K}_{g}^{*} \widetilde{K}_{g}\right)^{1 / 2}$, identifying the kernel $\widetilde{K}_{g}$ with the corresponding operator on $L^{2}(R) 。(3.1)$ and $(3.6)$ imply

$$
\begin{aligned}
& T\left\langle K_{g} \prod_{I} \exp \left[-\int_{I} B\left(\tau, s_{g}\right) d \tau\right]\right\rangle_{N} \\
& \quad \leqslant \sum_{\#}\left\langle\operatorname{Tr}\left|\widetilde{K}_{g}\right| \prod_{I} \exp \left[+\int_{I} d\left(\phi, \tau, s_{g}\right) d \tau\right]\right\rangle_{N} .
\end{aligned}
$$

When $\widetilde{K}_{g}$ is smeared by more than two $b^{\# \text {, }}$, it is a tensor product of two-fermion kernels as in (3.7). This is because any fermion graph is a collection of subgraphs which are either lines with two open ends or closed loops. The open lines correspond to two-fermion kernels, and the loops are functions on $Q$ space, which only contribute numerical factors at a given point in $Q$ space. Define the trace of a tensor product to be the product of the individual traces. The restriction to a finite number of modes is removed by a limiting argument. Therefore, the following lemma holds:
Lemma: Suppose $d\left(\phi, \tau, s_{g}\right)$ is a real-valued measurable function on $Q$ space and that, as operators,

$$
B\left(\tau, s_{g}\right) \geqslant-d\left(\phi, \tau, s_{g}\right) .
$$

Then

$$
\begin{aligned}
& T\left\langle K_{g} \prod_{I} \exp \left[-\int_{I} B\left(\tau, s_{g}\right) d \tau\right]\right\rangle_{N} \\
& \quad \leqslant\left\langle\operatorname{Tr}\left|\widetilde{K}_{g}\right| \prod_{I} \exp \left[\int_{I} d\left(\phi, \tau, s_{g}\right) d \tau\right]\right\rangle_{N}\left(\prod_{I} 2^{n(I)}\right) .
\end{aligned}
$$

Recall from $(2.21 \mathrm{a})$ that $n(I)$ is the number of vertices in interval $I$. The factor $2^{n(I)}$ overcounts sums over \#.

\section{CONVERGENCE OF THE EXPANSION}

In Sec. 7 , it is shown that, given $\epsilon>0, \exists d\left(\phi, \tau, s_{g}\right)$ as in Lemma (3.8)

$$
\begin{aligned}
& B\left(\tau, s_{g}\right) \geqslant-d\left(\phi, \tau, s_{g}\right), \\
& \left\langle\prod_{I} \exp \left[+2 \int_{I} d\left(\phi, \tau, s_{g}\right) d \tau\right]\right\rangle_{N} \leqslant \prod_{I}[O(1)]^{\rho^{(I)} \epsilon}
\end{aligned}
$$

$[\rho(I)$ is defined by $(2.17 \mathrm{a})] .(2.20),(4.1)$, Lemma (3.8), and the Cauchy-Schwartz inequality imply

$$
\left\langle e^{-T H}\right\rangle \leqslant \sum_{g} \int d s_{\xi}\left\langle\left(\operatorname{Tr}\left|\tilde{K}_{8}\right|\right)^{2}\right\rangle_{N}^{1 / 2}\left(\prod_{l}[O(1)]^{\left[n(I)+\rho(r)^{\epsilon}\right]}\right.
$$

Let $l$ be a line in a graph. Define

$$
\begin{aligned}
d_{l}= & \max \{1, \text { number of complete unit intervals that } l \\
& \text { crosses }\} .
\end{aligned}
$$

Let $\{g: n(I)\}$ denote the set of all $g$ with a given $n(I)$ specified for each $I$. The following estimate is used to count fermion graphs:

$$
\sum_{l g: n(I)\}}\left(\prod_{l} d_{l}^{-5}\right) \leqslant \prod_{I}[O(1)]^{n(I)}[2 n(I)] !
$$

The product is over all lines in the fermion graph, $F$, specified by $g$ [see (2.21a)]. A factor, $2^{n(I)}$, for each interval, $I$, overcounts the number of possible functions in $(2.21 \mathrm{a})$, part (2). A factor $O(1)^{n(I)}$ for each $I$ overcounts the number of ways in which $C$ vertices can have open lines. Therefore, the proof of (4.4) is reduced to showing that

$$
\sum_{G} \prod_{i \in G} d_{l}^{-5} \leqslant \prod_{I}\left([O(1)]^{n(I)}[2 n(I) !),\right.
$$

where the sum runs over all graphs with no open lines, which can be drawn on $\sum_{I} n(I)$ vertices, where the vertices are fixed in advance to have either one leg or two legs. This is proved by Dimock and Glimm, Lemma (2.6) in Ref. 6. (4.2), (4.4), and (2.17b) imply, for $\epsilon>0$,

$$
\begin{aligned}
\left\langle e^{-T H}\right\rangle \leqslant & \sum_{\{n(I)\}}\left(\prod_{I}[O(1)]^{\left(n(I)+n(I)^{\epsilon \alpha}\right.}[2 n(I)] !\right) \\
& \times \sup _{\{g: n(I)\}}\left\{\left(\prod_{l} d_{l}^{5}\right) \int d s_{\delta}\left\langle\left(\operatorname{Tr}\left|\tilde{K}_{\xi}\right|\right)^{2}\right\rangle_{N}^{1 / 2}\right\} .
\end{aligned}
$$

Define $d_{v}$ to be the sum of the contraction distances $d_{t}$ over lines $l$ that leave vertex $\nu$. Let $L_{\nu}$ be the lower momentum cutoff in $J_{\nu}\left(p_{1}, p_{2}\right)$ [see $(2.21 b)$ ]. In Secs. 5 and 6 the following lemma is proved.

$$
\begin{aligned}
& \text { Lemma: } \mathbf{3} \eta>0: \forall m \geqslant 0, \\
& \begin{array}{l}
\int d s_{g}\left\langle\left(\operatorname{Tr}\left|\tilde{K}_{g}\right|\right)^{2}\right\rangle_{N}^{1 / 2} \\
\quad \leqslant \prod_{I}\left(\left[O(1) \log ^{2} n(I)\right]^{n(I)}[n(I) !]^{2} \prod_{\nu \in I}\left[1+L_{\nu}\right]^{-\pi} d_{\nu}^{-m}\right) .
\end{array}
\end{aligned}
$$


The product over $\nu \in I$ means the product over all vertices in the interval $I$. (4.6) and (4.7) imply, for $\epsilon>0$ and for all $m \geqslant 0$,

$$
\begin{aligned}
\left\langle e^{-T H}\right\rangle \leqslant & \sum_{\{n(I)\}}\left(\prod_{I}\left[O(1) \log ^{2} n(I)\right]^{\left[n(I)+n(I)^{\epsilon \alpha}\right]}[n(I) !]^{4}\right) \\
& \times \sup _{\{g: n(I)\}}\left(\prod_{I} \prod_{\nu \in I}\left(1+L_{\nu}\right)^{-n} d_{\nu}^{-m}\right) .
\end{aligned}
$$

The $m$ has been relabelled to include the $d_{l}^{5}$ in (4.6). Recall from the description of the expansion in Sec. 2 that if $\nu$ is a $\mathrm{P}$ vertex formed during the $(n+1)$ th application of $(\mathrm{P})$ in a given interval $I$, then $L_{\nu}=K_{n}$ 。 If $\nu$ is a $\mathrm{C}$ vertex, $L_{\nu}=0$. By replacing $\eta$ by $\eta / 3$, if necessary, the factor $\left(1+L_{\nu}\right)$ in $(4.8)$ can be replaced by $\left(1+L_{\nu}^{\prime}\right)$, where $L_{\nu}^{\prime}=L_{\nu}$ if $\nu$ is a $\mathrm{P}$ vertex, but if $\nu$ is a $\mathrm{C}$ vertex, $L_{\nu}^{\prime}$ is the lower momentum cutoff of a $\mathrm{P}$ vertex contracted to $\nu$. If there are two such $\mathrm{P}$ vertices, then the largest lower momentum cutoff is chosen. The expansion is such that every $C$ vertex is contracted to a $P$ vertex, and, of course, no $P$ vertex can contract to more than $2 \mathrm{C}$ vertices.

For a fixed $I$ and $n(I)$, define $G(n(I))$ to be the set of all $g$ which have $n(I)$ vertices in $I_{\circ}(4.8)$ implies, for $\epsilon>0$ and all $m>0$,

$$
\begin{aligned}
\left\langle e^{-T H}\right\rangle \leqslant & \prod_{I}\left[\sum_{n(I)}\left[O(1) \log ^{2} n(I)\right]^{\left[n(I)+n(I)^{\epsilon \alpha}\right.}(n(I) !)^{4}\right. \\
& \left.\times \sup _{B \in G_{(n(I))}}\left(\prod_{\nu \in I}\left[1+L_{\nu}^{\prime}\right]^{-n} d_{\nu}^{-m}\right)\right] .
\end{aligned}
$$

In order to prove (1.2) and thereby bound (1.1) for the Fock vacuum, it suffices to show that, for sufficiently large $m$ and $\alpha$,

$$
\sup _{g \in \mathcal{G}_{(n(I))}}\left(\prod_{\nu \in I}\left(1+L_{\nu}^{\prime}\right)^{-\eta} d_{\nu}^{-m}\right) \leqslant O(1)[n(I) !]^{-5} .
$$

For, given $m$ and $\alpha$ such that (4.10) holds, choose $\epsilon$ small so that $\epsilon \alpha \leqslant 1$. Then (4.9) and (4.10) imply (1.2) for the Fock vacuum.

For the proof of $(4.10)$ the following notation is introduced. For a fixed interval $I, n(I)$, and $g \in G(n(I))$, define, for $i=2,3,4, \cdots, \mathrm{C}_{i}$ to be the set of all $\mathrm{C}$ vertices in $I$ which contract to a $\mathrm{P}$ vertex via a line $l$ with $d_{l}=i .\left[d_{l}\right.$ is defined by (4.3). $]$ Define $\mathrm{C}_{1}$ to be the union of the set of $\mathrm{P}$ vertices in $I$ with the set of all $\mathrm{C}$ vertices in $I$ which contract to a $\mathrm{P}$ vertex via a line $l$ with $d_{l}=1$. The fact that a $C$ vertex can contract to at most $2 \mathrm{P}$ vertices implies that

$$
\begin{aligned}
& \sup _{s \in G^{(n(I))}} \prod_{\nu \in I}\left(1+L_{\nu}^{\prime}\right)^{-n} d_{\nu}^{-m} \\
& \quad \leqslant \sup _{g_{\in} \in \mathcal{G}_{(n(I))}}\left(\prod_{i=1}^{\infty} \prod_{\nu \in C_{i}}\left(1+L_{\nu}^{\prime}\right)^{-\eta} d_{\nu}^{-m}\right)^{1 / 2} \\
& \leqslant \sup _{\xi_{\in} \in \mathcal{G}_{(n(I))}}\left(\prod_{i=1}^{\infty} i^{-m\left|C_{i}\right|} \prod_{\nu \in \mathrm{C}_{i}}\left(1+L_{\nu}^{\prime}\right)^{-\eta}\right)^{1 / 2},
\end{aligned}
$$

where $\left|\mathrm{C}_{i}\right|=$ number of elements in $\mathrm{C}_{i}$. Define $\widetilde{\mathrm{C}}_{i}$ to be the set of all $P$ vertices that are either in $C_{i}$ or contract to $\mathrm{C}$ vertices in $\mathrm{C}_{i}$. The definition of $L_{\nu}^{\prime}$ and the fact that at most two $\mathrm{P}$ vertices can contract to a given $\mathrm{C}$ vertex imply that

$$
\prod_{\nu \in \mathrm{C}_{i}}\left(1+L_{\nu}^{\prime}\right)^{-\eta} \leqslant\left(\prod_{\nu \in \tilde{C}_{i}}\left(1+L_{\nu}\right)^{-\eta}\right)^{1 / 2}
$$

The definition of $\tilde{\mathrm{C}}_{i}$ implies that there exists an interval, $I^{\prime}$, that contains at least $\left|C_{i}\right| / 12$ of the $\mathrm{P}$ vertices in $\widetilde{C}_{i}$. Since these $P$ vertices are in the same interval, as a consequence of the way in which the expansion is generated, they must all have different cutoffs, $L_{\nu}$, selected from the set $\left\{K_{1}, K_{2}, K_{3}, \cdots\right\}$. Recall from (1.9) that $K_{j}=j^{\alpha}$; therefore,

$$
\prod_{\nu \in \tilde{C}_{i}}\left(1+L_{\nu}\right)^{-\eta} \leqslant\left(\left|\mathrm{C}_{i}\right| / 12 !\right)^{-\eta \alpha} \text {. }
$$

(4.11), (4.12), and (4.13) imply that

$$
\begin{aligned}
& \sup _{\in \in \mathcal{G}(n(I))} \prod_{\nu \in I}\left(1+L_{\nu}^{\prime}\right)^{-n} d_{\nu}^{-m} \\
& \quad \sup _{g \in \mathcal{G}(n(I))} \prod_{i=1}^{\infty}\left[i^{-m\left|C_{i}\right| / 4}\left(\left|\mathrm{C}_{i}\right| / 12 !\right)^{-n \alpha / 4}\right],
\end{aligned}
$$

where $g \in G(n(I))$ implies that $\sum_{i}\left|C_{i}\right| \geqslant n(I)$; therefore, the right-hand side of (4.14) can be majorized by taking the supremum over $\left|C_{i}\right|$ such that

$$
\sum_{i}\left|\mathbf{C}_{i}\right|=n(I) \text {. }
$$

This is done by first taking the log and using Lagrange multipliers, i. e., maximize

$$
\begin{aligned}
J= & -\sum_{i=1}^{\infty}\left[m \frac{\left|\mathrm{C}_{\boldsymbol{i}}\right|}{4} \log i+\frac{\eta \alpha}{4}\left(\frac{\left|\mathrm{C}_{\boldsymbol{i}}\right|}{12}\right)\left(\log \frac{\left|\mathrm{C}_{\boldsymbol{i}}\right|}{12}-1\right)\right] \\
& +\lambda\left(n(I)-\sum\left|\mathrm{C}_{\boldsymbol{i}}\right|\right)
\end{aligned}
$$

By Sterling's formula, the right-hand side of (4.15) is majorized by the supremum of $e^{J}$. On differentiating, $(m / 4) \log i+(\eta \alpha / 48) \log \left(\left|\mathrm{C}_{i}\right| / 12\right)+\lambda=0$ for $i=1,2,3, \cdots$, $\sum_{i=1}^{\infty}\left|\mathrm{C}_{i}\right|=n(I)$.

When $(4.17)$ hold,

$$
J=\sum_{i=1}^{\infty}\left|\mathrm{C}_{i}\right|(\lambda+\eta \alpha / 48)=n(I)(\lambda+\eta \alpha / 48) .
$$

If $m / \alpha \geqslant 2 \eta / 12, \lambda$ can be eliminated from (4.18), by using (4.17), to show that

$$
J \leqslant-n(I)[(\eta \alpha / 48) \log n(I)-O(\eta \alpha)] .
$$

(4.19) and (4.14) imply (4.10).

This completes the proof of boundedness below for the Hamiltonian except for the estimates (4.1), which are proved in Sec. 7, and Lemma (4.7), which is proved in Secs. 5 and 6.

\section{ESTIMATE ON BOSON EXPECTATIONS}

In this section the left-hand side of (4.7) is estimated in terms of norms on kernels. The estimates on these norms, needed to complete the proof of Lemma (4.7), are given in Sec. 6. The method is based on techniques from Ref. 6. A more elegant, but less elementary, method relying on $L_{\phi}$ estimates and hypercontractivity is also outlined. This is taken from Ref. 7. The author is grateful to Ira Herbst for drawing his attention to the $L_{p}$ method.

Let $\lambda$ denote a vertex at time $s_{\lambda \text { 。 Let }} \bar{k}_{i}=\left(k_{i}^{0}, k_{i}^{1}\right)$ be Euclidean momenta. Define

$$
\begin{aligned}
H_{\lambda}\left(\bar{k}_{1}, \bar{k}_{2}\right)= & \int Q_{\lambda}\left(p_{1}, p_{2}, k_{1}^{1}\right) \bar{Q}_{\lambda}\left(p_{1}, p_{2}, k_{2}^{1}\right) d p_{1} d p_{2} \\
& \times \exp \left(i k_{1}^{0} s_{\lambda}\right) \exp \left(-i k_{2}^{0} s_{\lambda}\right),
\end{aligned}
$$


where $Q_{\lambda}$ was defined by $(2.33)$. Consider the special case in which $\widetilde{K}_{g}$ is the kernel of a two-particle fermion operator, as in (3.7); then the fact that the trace norm of a product of operators is majorized by the product of the Hilbert-Schmidt norms of the individual operators implies that

$$
\left(\operatorname{Tr}\left|\tilde{K}_{g}\right|\right)^{2} \leqslant \prod_{\lambda} \int H_{\lambda}\left(\bar{k}_{1}, \bar{k}_{2}\right) \phi\left(\bar{k}_{1}\right) \phi\left(-\bar{k}_{2}\right) d \bar{k}_{1} d \bar{k}_{2} .
$$

$\lambda$ runs over the vertices specified by $g$. (5.2) continues to hold when $\widetilde{K}_{g}$ is a tensor product of two-particle fermion operator kernels. Equivalently, one can say that (5.2) holds for any $g$ with a fermion graph $F$ [see (2.21a)] consisting of lines with open ends. In fact $F$ may also include nondivergent closed fermion loops, because these are traces of products of operators of the form implied by the right-hand side of (5.2). Therefore, a general $\tilde{K}_{g}$ can be estimated by

$$
\begin{aligned}
\left(\operatorname{Tr}\left|\tilde{K}_{g}\right|\right)^{2} \leqslant & \prod_{\lambda} \int H_{\lambda}\left(\bar{k}_{1}, \bar{k}_{2}\right) \phi\left(\bar{k}_{1}\right) \phi\left(-\bar{k}_{2}\right) d \bar{k}_{1} d \bar{k}_{2} \\
& \times \prod_{\mu}\left(\int R_{\mu}\left(\bar{k}_{1}, \bar{k}_{2}\right): \phi\left(\bar{k}_{1}\right) \phi\left(-\bar{k}_{2}\right): d \bar{k}_{1}, d \bar{k}_{2}+\delta E_{\mu}\right)^{2},
\end{aligned}
$$

where $\lambda$ runs over all vertices which are not part of cancelled renormalization subgraphs and $\mu$ runs over $P$ vertices which are part of cancelled renormalization subgraphs. $R_{\mu}$ and $\delta E_{\mu}$ are given by $(2.15)$ and $(2.16)$. (5.3) can be rewritten as

$$
\begin{aligned}
\left(\operatorname{Tr}\left|\tilde{K}_{s}\right|\right)^{2} \leqslant & \sum_{S} \underset{\substack{\mu_{1} \in s_{1}^{\lambda} \mu_{2} \in s^{c}\\
}}{\prod_{\lambda}}\left[\int H_{\lambda}\left(\bar{k}_{1}, \bar{k}_{2}\right) \phi\left(\bar{k}_{1}\right) \phi\left(-\bar{k}_{2}\right) d \bar{k}_{1} d \bar{k}_{2}\right] \\
& \times\left[\int R_{\mu_{1}}\left(\bar{k}_{1}, \bar{k}_{2}\right): \phi\left(\bar{k}_{1}\right) \phi\left(-\bar{k}_{2}\right): d \bar{k}_{1} d \bar{k}_{2}\right]\left[\delta E_{\mu_{2}}\right],
\end{aligned}
$$

where $S$ runs over subsets of the set, containing twice, $P$ vertices which are part of cancelled renormalization subgraphs. The complement of $S$ is with respect to this set.

$$
\begin{aligned}
& \text { Define } \\
& \qquad C(\bar{k})=\mathbf{1} /\left(m^{2}+|\bar{k}|^{2}\right) .
\end{aligned}
$$

This is the Fourier transform of the covariance operator of the free boson measure. The vacuum expectation, or alternatively the integral with respect to the free measure of the right-hand side of $(5.4)$, can be performed exactly so that

$$
\left\langle\left(\operatorname{Tr}\left|\tilde{K}_{\xi}\right|\right)^{2}\right\rangle_{N} \leqslant \sum \sum_{S} \int \prod_{\substack{\mu_{2} \in S^{c} \\ \mu_{1} \in S \\ \lambda}} H_{\lambda} R_{\mu_{1}} \delta E_{\mu_{2}} \prod_{l \in G} C\left(\bar{k}_{l}\right) d \bar{k}_{l},
$$

where $G$ is a graph on the vertices labelled by $\mu_{1}, \mu_{2}$, and $\lambda$. Vertices labelled by $\lambda$ have two legs which are allowed to contract to each other. Vertices labelled by $\mu_{1}$ have two legs which are restricted to contract to legs on other vertices. Vertices labelled by $\mu_{2}$ have no legs. $l$ runs over the lines in $G$ and $\bar{k}_{l}$ denotes the corresponding momenta. (More details are given in Ref. 6.) Let $\chi(t)$ be function in $C^{\infty}(R)$ such that $\chi(t) \equiv 1$, if $|t| \geqslant \frac{1}{2} ; \equiv 0$, if $|t| \leqslant \frac{1}{4} . d_{l}$ is defined as in (4.3). Define, for each $l$,

$$
\begin{array}{rlrl}
\chi_{l}(t) & \equiv 1, & & \text { if } d_{l}=1 \\
& \equiv \chi^{(}\left(t / d_{l}\right) & \text { if } d_{l}>1
\end{array}
$$

Regard $\chi_{l}$ as being a function of two variables, with $t$ being dual to $k_{0}$. Then, in the right-hand side of (5.6), $C\left(\bar{k}_{l}\right)$ can be replaced by $\left(\hat{\chi}_{l} * C\right)\left(\bar{k}_{l}\right)$ without changing its value, because vertices connected by lines with $d_{l}>1$ are localized in separated intervals in time. (There is no momentum cutoff on $k^{0}$ components.) With this replacement made, regard the right-hand side of (5.6) as a product of operators. For example, for a given vertex $\lambda$, the corresponding operator would have the kernel

$$
\left(\hat{\chi}_{l_{1}} * C\right)^{1 / 2}\left(\vec{k}_{1}\right) H_{\lambda}\left(\bar{k}_{1}, \bar{k}_{2}\right)\left(\hat{\chi}_{l_{2}} * C\right)^{1 / 2}\left(\vec{k}_{2}\right) \text {. }
$$

(It does not matter which square root is chosen.) Depending on how the lines $l_{1}$ and $l_{2}$ leave $\lambda$, this kernel is to be thought of as an operator either from $L^{2}\left(R^{2}\right)$ to $L^{2}\left(R^{2}\right)$ or from $L^{2}\left(R^{4}\right)$ to $\mathbb{C}$. [If $l_{1}$ and $l_{2}$ are the same line because $\lambda$ is contracted to itself, then it is not treated this way. In this case $\lambda$ gives rise to a constant. ] The right-hand side of (5.6) is now majorized by taking Hilbert-Schmidt norms of operators and absolute values of constants. (This is a simple version of techniques used by Glimm and Jaffe in Ref. 4。) The Hilbert-Schmidt norms are simplified by the estimate, for $n \geqslant 0$,

$$
\left|\left(\hat{\chi}_{l} * C\right)(\bar{k})\right| \leqslant O(1) d_{l}^{-n} C(\bar{k}) .
$$

The proof of (5.9) is postponed. The Hilbert-Schmidt norm corresponding to a vertex $\lambda$, is thereby less than $O(1) d_{l_{1}}^{-n} d_{l_{2}}^{-n}\left\|C^{1 / 2} H_{\lambda} C^{1 / 2}\right\|_{2}$, which is majorized by $O(1) d_{l_{1}}^{-n} d_{l_{2}}^{-n}$ $\times \operatorname{tr}\left(C^{1 / 2} H_{\lambda} C^{1 / 2}\right)$ because $C^{1 / 2} H_{\lambda} C^{1 / 2}$ is a positive operator on $L^{2}\left(R^{2}\right)$. These remarks prove that, for $n \geqslant 0$,

$$
\begin{aligned}
\left\langle\left(\operatorname{Tr}\left|\widetilde{K}_{g}\right|\right)^{2}\right\rangle_{N} \leqslant & \sum_{S} \prod_{\mu_{1}, \mu_{2}, \lambda}\left\|Q_{\lambda}\right\|_{C, 2}^{2}\left\|R_{\mu_{1}}\right\|_{C_{2} 2}\left|\delta E_{\mu_{2}}\right| \\
& \times \sum \prod_{G} \prod_{i \in G} O(1) d_{l}^{-n},
\end{aligned}
$$

where $\mu_{1}$ runs over $S$ and $\mu_{2}$ over $S^{c}$ and, by definition,

$$
\begin{aligned}
& \left\|Q_{\lambda}\right\|_{C_{2} 2}^{2}=\int\left|Q_{\lambda}\left(p_{1}, p_{2}, \bar{k}\right)\right|^{2} C(\bar{k}) d \bar{k} d p_{1} d p_{2}, \\
& \left\|R_{\mu_{1}}\right\|_{C_{2} 2}^{2}=\int\left|R_{\mu_{1}}\left(\bar{k}_{1}, \bar{k}_{2}\right)\right|^{2} C\left(\bar{k}_{1}\right) C\left(\bar{k}_{2}\right) d \bar{k}_{1} d \bar{k}_{2} .
\end{aligned}
$$

Note that $\left\|Q_{\lambda}\right\|_{C, 2}=\operatorname{tr}\left(C^{1 / 2} H_{\lambda} C^{1 / 2}\right)$ by (5.1). From Ref. 6, Lemma (2.6),

$$
\sum_{G} \prod_{i \in G} O(1) d_{i}^{-5} \leqslant \prod_{I} O(1)^{n(I)}[2 n(I)] ! ;
$$

therefore, (5.10) implies

$$
\begin{aligned}
\left\langle\left(\operatorname{Tr}\left|\tilde{K}_{g}\right|\right)^{2}\right\rangle_{N} \leqslant & \sum_{S} \prod_{\mu_{1}, \mu_{2, \lambda}}\left\|Q_{\lambda}\right\|_{C_{, 2}}^{2}\left\|R_{\mu_{1}}\right\|_{C, 2}\left|\delta E_{\mu_{2}}\right| \\
& \times \prod_{I} O(1)^{n(I)}[2 n(I)] !
\end{aligned}
$$

The proof of Lemma (4. 7) will now follow from estimates on the norms in (5.13). These are given in the next section.

$$
\begin{aligned}
& \text { (5.9) is proved by } \\
& \left\|C^{-1}\left(\hat{\chi}_{3} * C\right)\right\|_{\infty} \leqslant\left\|\left(m^{2}-\Delta\right) \chi_{l} \hat{C}\right\|_{1} \leqslant O(1) d_{l}^{-n},
\end{aligned}
$$

where the last inequality follows from the exponential decay of $\hat{C}$ away from the origin.

The $L_{p}$ method is now given. Suppose $f_{i}$ are two particle polynomials on Nelson space, whose time supports are localized in unit intervals denoted by $I$ in the usual way. Suppose $n(I) f_{i}$ 's are localized in $I$ in this manner: 


$$
\begin{aligned}
\left\langle\prod_{i} f_{i}\right\rangle_{N} & =\left\langle\prod_{i} \prod_{i \in I} f_{i}\right\rangle_{N} \\
& =\left\langle\prod_{I}\left[E_{I+1}\left(\prod_{i \in I} f_{i}\right) E_{I}\right]\right\rangle_{N},
\end{aligned}
$$

where $E_{I}$ denotes the projection onto the time slice $t=I$ in Nelson space. This is not to be confused with the projection onto the interval $I$. This notation is described in Ref. 7:

$$
\leqslant \prod_{I}\left\|E_{I+1}\left(\underset{i \in I}{\prod_{I}} f_{i}\right) E_{I}\right\|
$$

The bars denote the operator norm on $L^{2}(Q)$, i. e., Nelson space. By hypercontractivity (see Chap. III, Ref. $7),(5.15)$ is overestimated by

$$
\prod_{i}\left\|\prod_{i \in I} f_{i}\right\|_{i}
$$

provided $p$ sufficiently large. Applying the Hölder inequality to $(5.16)$ implies

$$
\left\langle\prod_{i} f_{i}\right\rangle \leqslant \prod_{I} \prod_{i \in I}\left\|f_{i}\right\|_{p n(I)}
$$

Since $f_{i}$ is a two-particle operator for each $i$, Nelson's best estimate applied to the operator $\exp (-t N)$ for sufficiently large $t$ shows that

$$
\left\|f_{i}\right\|_{p n_{I}} \leqslant(p n(l)-1)\left\|f_{i}\right\|_{2} \text {. }
$$

Nelson's best estimate is also described in Ref. 7, Chap. III. $(5.17)$ and $(5.18)$ imply that

$$
\left\langle\prod_{i} f_{i}\right\rangle \leqslant \prod_{I}\left(O(1)^{n(I)} n(I) ! \prod_{i \in I}\left\|f_{i}\right\|_{2}\right)
$$

(5.19) applied to (5.4) implies (5.13).

\section{ESTIMATES ON KERNELS}

In this section the five estimates $(6.1)-(6.5)$ given below, are proved. For (6.1), suppose $\lambda$ is a completely contracted vertex, i. e., in the fermion graph $F$, neither of the lines $l_{1}$ and $l_{2}$ which leave $\lambda$ are open. Then, for $n \geqslant 0$ and some $\eta>0$ independent of $n$,

$$
\left\|Q_{\lambda}\right\|_{c_{2}} \leqslant O(\mathbf{1})\left(1+L_{\lambda}\right)^{-n} d_{i_{1}}^{-n} d_{i_{2}}^{-n} t_{l_{1}}^{-5 / 16} t_{i_{2}}^{-5 / 16},
$$

where $L_{\lambda}$ is the low momentum cutoff associated with $\lambda\left(L_{\nu} \neq 0\right.$ only for $\mathrm{P}$ vertices). $t_{t}$ is defined by (2. 22). For (6.2), suppose $\lambda$ is not completely contracted so that $l_{1}$ is an open line in $F$. Then, for $n \geqslant 0$,

$$
\left\|Q_{\lambda}\right\|_{C_{2} 2} \leqslant O(1)\left(1+L_{\lambda}\right)^{-n} \log ^{2} n(I)^{\alpha} d_{t_{2}}^{-n} t_{l_{1}}^{-1 / 16} t_{i_{2}}^{-1 / 2} \text {. }
$$

The factor $\left(1+L_{\lambda}\right)^{-n}$ is put in merely to make the notation uniform. In fact, since $\lambda$ has an open line, $\lambda$ is a $C$ vertex and therefore $L_{\lambda}=0$. For (6.3) and (6.4) suppose $\mu_{1}$ and $\mu_{2}$ are $P$ vertices in cancelled renormalization subgraphs; then

$$
\begin{aligned}
& \left\|R_{\mu_{1}}\right\|_{c_{2} 2} \leqslant O(1)\left(1+L_{\mu_{1}}\right)^{-\eta}\left(I+1-s_{\mu_{1}}\right)^{-1 / 32}, \\
& \left|\delta E_{\mu_{2}}\right| \leqslant O(1)\left(1+L_{\mu_{2}}\right)^{-\eta}\left(I+1-s_{\mu_{2}}\right)^{-1 / 32,}
\end{aligned}
$$

where $s_{\mu_{1}}$ and $s_{\mu_{2}}$ are the times of the $P$ vertices and $I$, as usual, is the integer that labels the unit interval containing $\mu_{1}\left(\mu_{2}\right)$. For (6.5) the notation $I(\mu)$ is used to indicate the interval containing a vertex $\mu$ and $\mu$ runs over all $\mathrm{P}$ vertices which are part of cancelled renormalization subgraphs:

$$
\int d s_{g} \prod_{l \in F} t_{l}^{-\sigma_{l}} \prod_{\mu}\left(I(\mu)+1-s_{\mu}\right)^{-1 / 32} \leqslant \prod_{I} O(1)^{n(I)} n(I) !,
$$

where if $l$ is open, $\sigma_{l}=\frac{1}{16}$; if $l$ connects two completely contracted vertices, $\sigma_{l}=\frac{10}{16}$; if $l$ connects to a vertex which is not completely contracted, $\sigma_{l}=\frac{13}{16}$. The case in which $l$ connects two vertices which are both not completely contracted cannot occur by the way the expansion is defined.

Lemma (4.7) is proved by combining (6.1)-(6.5) with (5.13) and noting that the number of subsets $S$ summed over in $(5.13)$ is less than $\Pi_{I} 2^{n(n)}$.

Proof of (6.1): from (2.23b), (1.8), and (5.11), for $n \geqslant 0$,

$$
\begin{aligned}
\left\|Q_{\lambda}\right\|_{C, 2}^{2} \leqslant & O(1) \int\left|\hat{g}\left(p_{1}+p_{2}+k^{1}\right)\right|^{2} J_{\lambda}\left(p_{1}, p_{2}\right) \exp \left(-\omega_{1} t_{l_{1}}\right) \\
& \times \exp \left(-\omega_{2} t_{l_{2}}\right) C(\bar{k}) d p_{1} d p_{2} d \bar{k} \\
\leqslant & O(1) \int\left|\hat{g}\left(p_{1}+p_{2}+k^{1}\right)\right|^{2} J_{\lambda}\left(p_{1}, p_{2}\right) \omega_{1}^{-10 / 16} \omega_{2}^{-10 / 16} \\
& \times \mu^{-1}\left(k^{1}\right) d p_{1} d p_{2} d k^{1} d_{i_{1}}^{-2 n} d_{l_{2}}^{-2 n} t_{l_{1}}^{-10 / 16} t_{l_{2}}^{-10 / 16}
\end{aligned}
$$

$J_{\lambda}$ contains a lower cutoff at $L_{\lambda}$; therefore,

$$
\begin{aligned}
\left\|Q_{\lambda}\right\|_{C, 2}^{2} \leqslant & O(1) \int_{\substack{\left|p_{1}\right| \geqslant L_{\lambda} \\
\text { or } \\
\left|p_{2}\right| \geqslant L_{\lambda}}}\left|\hat{g}\left(p_{1}+p_{2}+k^{1}\right)\right|^{2} \omega_{1}^{-10 / 16} \omega_{2}^{-10 / 16} \\
& \times \mu^{-1}\left(k^{1}\right) d p_{1} d p_{2} d k^{1} d_{l_{1}}^{-2 n} d_{l_{2}}^{-2 n} t_{l_{1}}^{-10 / 16} t_{l_{2}}^{-10 / 16} \\
\leqslant & O(1)\left(1+L_{\lambda}\right)^{-2 n} d_{l_{1}}^{-2 n} d_{l_{2}}^{-2 n} t_{l_{1}}^{-10 / 16} t_{l_{2}}^{-10 / 16}
\end{aligned}
$$

(1.4) is used to obtain the last inequality. $\eta$ is some small number $>0$.

Proof of (6.2): from (2.23c), (1.8), (5.11), and the fact that $\rho(I) \leqslant n(I)^{\alpha}$ [see (2.17) for the definition of $\rho(I)]$

$$
\begin{aligned}
& \left\|Q_{\lambda}\right\|_{C}^{2} \leqslant O(1) \int_{\left|p_{1}\right| \leqslant n(I)^{\alpha}}\left|\hat{g}\left(p_{1}+p_{2}+k^{1}\right)\right|^{2} J_{\lambda}\left(p_{1}, p_{2}\right) \\
& \times \exp \left(-\omega_{2} t_{l_{2}}\right) C(\bar{k}) d p_{1} d p_{2} d \bar{k} \\
& +O(1) \int_{\left|p_{1}\right| \geqslant n(I)} \alpha\left|\hat{g}\left(p_{1}+p_{2}+k^{1}\right)\right|^{2} J_{\lambda}\left(p_{1}, p_{2}\right) \\
& \times \exp \left(-\omega_{1} t_{l_{1}}\right) \exp \left(-\omega_{2} t_{t_{2}}\right) C(\bar{k}) d p_{1} d p_{2} d \bar{k} \\
& \leqslant O(1) \int_{\left|p_{1}\right| \leqslant n(I)^{\alpha}}\left|\hat{g}\left(p_{1}+p_{2}+k^{1}\right)\right|^{2} \omega_{2}^{-1} \mu\left(k^{1}\right)^{-1} d p_{1} d p_{2} d k^{1} \\
& \times d_{l_{2}}^{-2 n} t_{l_{2}}^{-1}+O(1) \int_{\left|p_{1}\right| \geqslant n(I)}{ }^{\alpha}\left|\hat{g}\left(p_{1}+p_{2}+k^{1}\right)\right|^{2} \omega_{2}^{-1} \omega_{1}^{-1 / 8} \\
& \times \mu\left(k^{1}\right)^{-1} d p_{1} d p_{2} d k^{1} d_{l_{2}}^{-2 n} t_{l_{2}}^{-1} t_{l_{1}}^{-1 / 8} \text {. }
\end{aligned}
$$

The proof of (6.2) is completed by the elementary estimates:

$$
\begin{aligned}
& \int_{\left|p_{1}\right| \leqslant n(I)^{\alpha}}\left|\hat{g}\left(p_{1}+p_{2}+k^{1}\right)\right|^{2} \omega_{2}^{-1} \mu\left(k^{1}\right)^{-1} d p_{1} d p_{2} d k^{1} \\
& \quad \leqslant O(1) \log ^{2} n(I)^{\alpha}, \\
& \int_{\left|p_{1}\right| \geqslant n()^{\alpha}}\left|\hat{g}\left(p_{1}+p_{2}+k^{1}\right)\right|^{2} \omega_{2}^{-1} \omega_{1}^{-1 / 8} \mu\left(k^{1}\right)^{-1} d p_{1} d p_{2} d k^{1} \\
& \quad \leqslant O(1) .
\end{aligned}
$$

Proof of (6.3): By (2.15) and the triangle inequality it is sufficient to prove (6.3) with $R_{\mu_{1}}$ replaced in turn by the following three kernels:

$$
\begin{aligned}
& \int_{\substack{\left|p_{1}\right| \leqslant K \\
\left|p_{2}\right| \leqslant K}} d p_{1} d p_{2} W_{p}\left(p_{1}, p_{2}, k_{1}^{1}\right) \bar{W}_{p}\left(p_{1}, p_{2}, k_{2}^{1}\right) \rho\left(p_{1}, p_{2}\right) \\
& \quad \times \frac{\exp \left[-\left(\omega_{1}+\omega_{2}-i k_{1}^{0}\right)\left(I+1-s_{\mu_{1}}\right)\right]}{\omega_{1}+\omega_{2}-i k_{1}^{0}} \exp \left[i\left(k_{1}^{0}-k_{2}^{0}\right) s_{\mu_{1}}\right]
\end{aligned}
$$




$$
\begin{aligned}
& \int_{\substack{\left|p_{1}\right| \leqslant K \\
\left|p_{2}\right| \leqslant K}} d p_{1} d p_{2} W_{p}\left(p_{1}, p_{2}, k_{1}^{1}\right) \bar{W}_{p}\left(p_{1}, p_{2}, k_{2}^{1}\right) \rho\left(p_{1}, p_{2}\right) \\
& \quad \times\left(\frac{1}{\omega_{1}+\omega_{2}-i k_{1}^{0}}-\frac{1}{\omega_{1}+\omega_{2}}\right) \exp \left[i\left(k_{1}^{0}-k_{2}^{0}\right) s_{\mu_{1}}\right] \\
& \int_{\substack{\left|p_{1}\right| \leqslant K \\
\left|p_{2}\right| \leqslant K}} d p_{1} d p_{2}\left(W_{p}\left(p_{1}, p_{2}, k_{1}^{1}\right) \bar{W}_{p}\left(p_{1}, p_{2}, k_{2}^{1}\right) \rho\left(p_{1}, p_{2}\right)\right. \\
& \left.\left.\quad \times \frac{1}{\omega_{1}+\omega_{2}} \exp \left[i\left(k_{1}^{0}-k_{2}^{2}\right) s_{\mu_{1}}\right)\right]\right)+\frac{\frac{1}{2} \delta m_{\mu_{1}}^{2}}{4 \pi} \hat{g} * \hat{g}\left(k_{1}^{\prime}-k_{2}^{\prime}\right) \\
& \quad \times \exp \left[i\left(k_{1}^{0}-k_{2}^{0}\right) s_{\mu_{1}}\right]
\end{aligned}
$$

where $\rho\left(p_{1}, p_{2}\right)$ is a lower cutoff at $L_{\mu_{1}}$ [see (1.10)]. These corresponding inequalities are respectively implied by $(6.15)-(6.17)$ below. For $\epsilon>0, \exists \eta>0$ such that for all $K$

$$
\begin{aligned}
& \iint_{p_{1}+p_{2}=\xi} \rho\left(p_{1}, p_{2}\right) \frac{\exp \left[-\left(\omega_{1}+\omega_{2}-i k_{1}^{0}\right)\left(I+1-s_{\mu_{1}}\right)\right]}{\omega_{1}+\omega_{2}-i k_{1}^{0}} \\
& \left|p_{1}\right|,\left|p_{2}\right| \leqslant K \\
& \leqslant O(1)\left(1+L_{\mu_{1}}\right)^{-n}\left(I+1-s_{\mu_{1}}\right)^{-1 / 32}, \\
& \int_{\substack{p_{1}+p_{2}=\xi \\
\left|p_{1}\right|,\left|p_{2}\right| \leqslant K}} \rho\left(p_{1}, p_{2}\right)\left|\frac{1}{\omega_{1}+\omega_{2}-i k_{1}^{0}}-\frac{1}{\omega_{1}+\omega_{2}}\right| \\
& \leqslant O(1)\left(1+L_{\mu_{1}}\right)^{-\pi}\left|k_{1}^{0}\right|^{6}, \\
& \mid \int_{p_{1}+p_{2}=\xi} \frac{\omega_{1} \omega_{2}-p_{1} p_{2}-M^{2}}{\omega_{1} \omega_{2}} \rho\left(p_{1}, p_{2}\right) \frac{1}{\omega_{1}+\omega_{2}} \\
& \left|p_{1}\right|,\left|p_{2}\right| \leqslant K \\
& -\int_{\substack{p_{1}+p_{2}=0 \\
\left|p_{1}\right|,\left|p_{2}\right| \leqslant K}} \frac{\omega_{1} \omega_{2}-p_{1} p_{2}-M^{2}}{\omega_{1} \omega_{2}} \rho\left(p_{1} p_{2}\right) \frac{1}{\omega_{1}+\omega_{2}} \mid \\
& \leqslant O(1)\left(1+L_{u_{1}}\right)^{-\eta}|\xi|^{\varepsilon} \text {. }
\end{aligned}
$$

For example, by $(6.17)$ the absolute value of $(6.14)$ is majorized by

$$
\begin{aligned}
& O(1)\left(1+L_{\mu_{1}}\right)^{-\eta} \int d \xi\left|\hat{g}\left(k_{1}^{1}-\xi\right)\right||\xi|^{\epsilon}\left|\hat{g}\left(\xi-k_{2}^{1}\right)\right| \\
& \leqslant \\
& O(1)\left(1+L_{\mu_{1}}\right)^{-\eta} \mu^{\epsilon / 2}\left(k_{1}^{1}\right) \mu^{\epsilon / 2}\left(k_{2}^{1}\right) \int d \xi \\
& \quad \times\left|\mu^{\epsilon / 2}\left(k_{1}^{1}-\xi\right) \hat{g}\left(k_{1}^{1}-\xi\right)\right|\left|\mu^{\epsilon / 2}\left(\xi-k_{2}^{1}\right) \hat{g}\left(\xi-k_{2}^{1}\right)\right| .
\end{aligned}
$$

Therefore, the $(C, 2)$ norm of $(6.14)$ is majorized by

$$
\begin{aligned}
& O(1)\left(1+L_{\mu_{1}}\right)^{-\eta}\left\{\int d \bar{k}_{1} d \bar{k}_{2}\left[\mu^{\epsilon / 2}|\hat{g}| * \mu^{\varepsilon / 2}|\hat{g}|\left(k_{1}^{1}-k_{2}^{1}\right)\right]^{2}\right. \\
& \left.\times \mu^{\varepsilon / 2}\left(k_{1}^{1}\right) C\left(\bar{k}_{1}\right) \mu^{\epsilon / 2}\left(k_{2}^{1}\right) C\left(\bar{k}_{2}\right)\right\}^{1 / 2} .
\end{aligned}
$$

By doing the integrals over $k_{1}^{0}$ and $k_{2}^{0}$ and changing the integration variables $(6.20)$ is less than

$$
\begin{aligned}
& O(1)\left(1+L_{\mu_{1}}\right)^{-\eta}\left\{\int d \eta\left[\mu^{\epsilon / 2}|\hat{g}| * \mu^{\epsilon / 2}|\hat{g}|(\eta)\right]^{2}\right. \\
& \left.\times \int_{k_{1}^{\prime}-\hbar \xi_{2=\eta}} \mu^{-1+\epsilon / 2}\left(k_{1}^{\prime}\right) \mu^{-1+\epsilon / 2}\left(k_{2}^{\prime}\right)\right\}^{1 / 2} \\
& \quad \leqslant O(1)\left(1+L_{\mu_{1}}\right)^{-\eta}\left\|\mu^{\epsilon / 2}|\hat{g}| * \mu^{\epsilon / 2}|\hat{g}|\right\|_{2} \\
& \quad=O(1)\left(1+L_{\mu_{1}}\right)^{-\eta}\left\|\left[\left(\mu^{\epsilon / 2}|\hat{g}|\right)^{\vee}\right]^{2}\right\|_{2} .
\end{aligned}
$$

In the last equality " $v$ " denotes the inverse Fourier transform. By the Tichmarsh theorem $(6.23)$ is majorized by

$$
O(1)\left(1+L_{\mu_{1}}\right)^{-\pi}\left\|\mu^{\epsilon / 2}|\hat{g}|\right\|_{4 / 3}^{2} \leqslant O(1)\left(1+L_{\mu_{1}}\right)^{-\pi}
$$

by (1.4). This completes the proof that, given (6.17), (6. 3) holds with $R_{\mu}$ replaced by $(6.14)$. In a similar way (6.15) and (6.16) imply that (6.3) holds with $R_{\mu_{1}}$ replaced by (6.12) and (6.13) respectively. Therefore, the proof of $(6.3)$ reduces to proving $(6.15)-(6.17)$. (6.15) and (6.16) are elementary. (6.17) is implied by the following three elementary inequalities. For $\epsilon>0$, $\mathbf{3} \eta>0$ such that

$$
\begin{aligned}
& \int d p\left|\frac{\omega(p) \omega(\xi-p)-p(\xi-p)-M^{2}}{\omega(p) \omega(\xi-p)}-\frac{\omega^{2}(p)+p^{2}-M^{2}}{\omega^{2}(p)}\right| \\
& \quad \times \rho(p, \xi-p) \frac{1}{\omega(p)+\omega(\xi-p)} \leqslant O(1)\left(1+L_{\mu_{1}}\right)^{-\eta}|\xi|^{\epsilon},(6 \\
& \int d p\left|\frac{1}{\omega(p)+\omega(\xi-p)}-\frac{1}{2 \omega(p)}\right| \rho\left(p_{1}, \xi-p\right) \\
& \leqslant O(1)\left(1+L_{\mu_{1}}\right)^{-\eta}|\xi|^{\epsilon}, \\
& \int d p \frac{1}{\omega(p)}\left|\chi_{l}(p)-\chi_{0}(p)\right| \leqslant O(1)\left(1+L_{\mu_{1}}\right)^{-\eta}|\xi|^{\epsilon},
\end{aligned}
$$

where $\chi_{\xi}(p)$ is the characteristic function of the set $\{|p| \leqslant K,|\xi-p| \leqslant K\} \cap\left\{\right.$ either $|p| \geqslant L_{\mu_{1}}$ or $\left.|\xi-p| \geqslant L_{\mu_{1}}\right\}$ and $\chi_{0}$ is given by putting $\xi=0$. This completes the proof of (6.3).

Proof of (6.4): From (2.16), (2.13), and (1.8)

$$
\begin{aligned}
& \delta E_{\mu_{2}}=\left(\frac{\lambda}{4 \pi}\right)^{2} \int_{\substack{\left|p_{1}\right| \leqslant k \\
\left|p_{2}\right| \leqslant K}}\left|\hat{g}\left(p_{1}+p_{2}+k\right)\right|^{2} \frac{\omega_{1} \omega_{2}-p_{1} p_{2}-M^{2}}{\omega_{1} \omega_{2}} \\
& \times \rho\left(p_{1}, p_{2}\right) \mu^{-1}(k) \frac{\exp \left\{-\left[\omega_{1}+\omega_{2}+\mu(k)\right]\left(I+1-s \mu_{2}\right)\right\}}{\omega_{1}+\omega_{2}+\mu(k)} \\
& \times d p_{1} d p_{2} d k \\
& \leqslant O(\mathbf{1}) \int d \xi|\hat{g}(\xi)|^{2} \int_{p_{1}+p_{2}+k=\xi} \\
& \quad \times \frac{\exp \left\{-\left[\omega_{1}+\omega_{2}+\mu(k)\right]\left(I+1-s_{\mu_{2}}\right)\right\}}{\omega_{1}+\omega_{2}+\mu(k)} \frac{1}{\mu(k)} \rho .
\end{aligned}
$$

(6.4) now follows from (1.4) and

$$
\begin{aligned}
& \int_{p_{1}+p_{2}+k=\xi} \frac{\exp \left\{-\left[\omega_{1}+\omega_{2}+\mu(k)\right]\left(I+1-s_{\mu_{2}}\right)\right\}}{\omega_{1}+\omega_{2}+\mu(k)} \frac{\rho}{\mu(k)} \\
& \leqslant O(1)\left(1+L_{\mu_{2}}\right)^{-\eta}\left(I+1-s_{\mu_{2}}\right)^{-1 / 32}
\end{aligned}
$$

Proof of (6.5): From (2.25) $\mathrm{P}$ vertices are time ordered, but $\mathrm{C}$ vertices are not. First assume that all vertices are time ordered and labelled in that order by $\nu=1,2,3, \cdots$. By $(2.25)$ each vertex is also restricted to lie in a certain interval. Suppose, for example, that a vertex $\nu$ in $[I-1, I]$ is contracted by a line $l$ to $\nu^{\prime \prime}$ in $[I, I+1]$. Let $s_{\nu} \leqslant s_{\nu+1} \leqslant \cdots s_{\nu^{\prime}} \leqslant I \leqslant s_{\nu^{\prime}+1} \leqslant \cdots \leqslant s_{\nu^{\prime \prime}}$ be the times of the intervening vertices. Then, for $\sigma_{\nu}, \sigma_{\nu+1} \ldots$ $\sigma_{\nu^{\mu}-1}$ positive numbers with $\sigma_{\nu}+\sigma_{\nu+1}+\cdots+\sigma_{\nu^{\prime \prime}-1}=\sigma_{l}$,

$$
\begin{aligned}
& t^{-\sigma_{l}}=\left(s_{\nu^{\prime \prime}}-s_{\nu}\right)^{-\sigma_{l}} \leqslant\left(s_{\nu+1}-s_{\nu}\right)^{-\sigma_{\nu}}\left(s_{\nu+2}-s_{\nu+1}\right)^{-\sigma_{\nu+1}}
\end{aligned}
$$

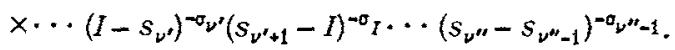

By use of estimates similar to $(6,30)$ it follows that

$$
\begin{aligned}
& \int_{\text {time ordered }} d s_{g} \prod_{l \in F} t_{l}^{-\sigma_{l}} \prod_{\mu}\left(I(\mu)+1-s_{\mu}\right)^{-1 / 32} \\
& \leqslant \underset{I}{\leqslant}\left[\int_{i=n(I)+1} \prod_{i=1} \frac{d t_{i}}{t_{i}^{31 / 32}} \delta\left(\sum_{i=1}^{n(I)+1} t_{i}-1\right)\right]
\end{aligned}
$$




$$
=\prod_{I} \frac{\Gamma(1 / 32)^{n(n)}}{\Gamma\left(\sum_{I} n(I) / 32\right)} \leqslant \prod_{I} O(1)^{n(t)}
$$

where the $\sigma_{l}$ are chosen as in (6.5). There are less than $\Pi_{I} n(I) !$ ways of time ordering the region of integration on the right-hand side of (6.5); therefore, (6.5) follows from (6.32).

\section{ESTIMATES ON THE EXPONENT}

In this section it is proved that, given $K$ and $\epsilon>0$ there exists a function $c(\phi)$ such that

$$
\begin{aligned}
& H_{0 F}+V+c(\phi) \geqslant-O\left(K^{5}\right), \\
& H_{0 B}-2 c(\phi) \geqslant-O(1) .
\end{aligned}
$$

A corresponding estimate is also proved for $G Y_{2}$. This proves the claim made in (2.1). By replacing $K$ with $K_{i}$ and referring to (2.24), (2.8), and (2.17a), the proof of (4.1) also follows.

\section{Define the quantities}

$$
\begin{aligned}
N_{\tau F} & =\int \omega^{\tau}(p)\left[b^{*}(p) b(p)+b^{* *}(p) b^{\prime}(p)\right] d p \text { on } \Omega^{\perp} \\
& =1 \text { on } \Omega, \\
N_{\tau B} & =\int \mu^{\top}(k) a^{*}(k) a(k) d k \text { on } \Omega^{\perp} \\
& =1 \text { on } \Omega .
\end{aligned}
$$

$\Omega$ is the Fock vacuum.

$$
\begin{aligned}
r\left(p_{1}, p_{2}\right)= & \text { characteristic function of the set } \\
& \left\{\left|p_{1}\right| \geqslant L \text { or }\left|p_{2}\right| \geqslant L\right\} .
\end{aligned}
$$

$L$ will be chosen later.

$$
\begin{aligned}
V_{p r}= & \int_{\left|p_{1}\right|,\left|p_{2}\right| \leqslant K}\left[b^{*}\left(p_{1}\right) b^{\prime *}\left(p_{2}\right)+b\left(-p_{1}\right) b^{\prime}\left(-p_{2}\right)\right] r\left(p_{1}, p_{2}\right) \\
& \times W_{p}\left(p_{1}, p_{2}, k\right) \phi(k) d p_{1} d p_{2} d k-\frac{1}{2}\left(\delta m_{K}^{2}-\delta m_{L}^{2}\right) \\
& \times \int g^{2}(x): \phi^{2}(x): d x-\left(E_{K}-E_{L}\right), \\
V_{s r}= & \int_{\left|p_{1}\right|,\left|p_{2}\right| \leqslant K}\left[b^{*}\left(p_{1}\right) b\left(-p_{2}\right)+b^{\prime *}\left(p_{1}\right) b^{\prime}\left(-p_{2}\right)\right] r\left(p_{1}, p_{2}\right) \\
& \times W_{s}\left(p_{1}, p_{2}, k\right) \phi(k) d p_{1} d p_{2} d k \\
\Gamma V_{p r}= & \int_{\left|p_{1}\right|,\left|p_{2}\right| \leqslant K}\left[b^{*}\left(p_{1}\right) b^{\prime *}\left(p_{2}\right)-b\left(-p_{1}\right) b^{\prime}\left(-p_{2}\right)\right] \frac{1}{\tilde{\omega}_{1}+\tilde{\omega}_{2}} \\
& \times r\left(p_{1}, p_{2}\right) W_{p}\left(p_{1}, p_{2}, k\right) \phi(k) d p_{1} d p_{2} d k,
\end{aligned}
$$

where $\tilde{\omega}(p)=\omega(p)-\omega^{\top}(p), \tau<1$. In order that $\tilde{\omega}>0$, assume the fermion mass is larger than unity. This is not an essential restriction. Note that $\Gamma V_{p r}$ is an antisymmetric operator designed so that

$$
\begin{aligned}
& {\left[\Gamma V_{p r}, H_{0 F}-N_{\tau F}\right]+V_{p r}+\frac{1}{2}\left(\delta m_{K}^{2}-S m_{L}^{2}\right)} \\
& \quad \times \int g^{2}(x): \phi^{2}(x): d x+\left(E_{K}-E_{L}\right)=0 .
\end{aligned}
$$

$(7.1 \mathrm{a}, \mathrm{b})$ are obtained by adding the inequalities $(7.8)$ given below.

Given $\tau \in(0,1)$, for sufficiently large $L$, there exists $c_{1}(\phi)$ such that

$$
\begin{aligned}
& H_{0 F}-N_{\tau F}+V_{p F}+c_{1}(\phi) \geqslant-O(\log K), \\
& \frac{1}{3} H_{0 B}-2 c_{1}(\phi) \geqslant 0 .
\end{aligned}
$$

Given $\epsilon>0$, for $L$ sufficiently large and $\tau$ close enough to $1(\tau<1)$, there exists $c_{2}(\phi)$ such that

$$
\frac{1}{2} N_{\tau F}+V_{s r}+c_{2}(\phi) \geqslant-O\left(K^{\epsilon}\right),
$$

$$
\frac{1}{3} H_{0 B}-2 c_{2}(\phi) \geqslant 0 .
$$

Given $L$ there exists $c_{3}(\phi)$ such that

$$
\begin{aligned}
& \frac{1}{2} N_{\tau F}+V_{L}+c_{3}(\phi) \geqslant-O(1), \\
& \frac{1}{3} H_{0 B}-2 c_{3}(\phi) \geqslant-O(1),
\end{aligned}
$$

where $V_{L}$ is defined by replacing $K$ by $L$ in the definition of $V$ in (1.6).

A proof for $(7.8 \mathrm{e}, \mathrm{f})$ may easily be constructed using the following remarks. (1) The mass counterterm in $V_{L}$ is formally positive. (2) Each kernel of $V_{L}$ can be written as the sum of products of Hermite functions plus remainder in such a way that the term corresponding to the remainder can be estimated by an $N_{\text {TF }}$ estimate as will be demonstrated for $(7.8 \mathrm{c}, \mathrm{d})$. The other term can be majorized by a suitable boson function by

$$
\left|\sum W_{i j k} b^{\#}\left(h_{i}\right) b^{\#}\left(h_{j}\right) \phi\left(h_{k}\right)\right| \leqslant \sum\left|W_{i j k}\right|\left|\phi\left(h_{k}\right)\right|,
$$

where $h_{i}$ are Hermite functions with unit $L_{2}$ norm.

(7. 8a, b) will now be proved by Glimm's dressing transformation (Ref, 1).

Define

$$
\tilde{b}(p)=b(p)-\left[\Gamma V_{p r}, b(p)\right], \quad \tilde{b}^{\prime}(p)=b^{\prime}(p)-\left[\Gamma V_{p r}, b^{\prime}(p)\right]
$$

and calculate, using (7.7), the operator $\int \tilde{\omega}(p)\left[\tilde{b}^{*}(p) \tilde{b}(p)\right.$ $\left.+\widetilde{b}^{\prime *}(p) \widetilde{b}^{\prime}(p)\right] d p$. Then, since this operator is positive,

$$
\begin{aligned}
0 \leqslant & H_{0 F}-N_{\tau F}+V_{p r}+\frac{1}{2}\left(\delta m_{K}^{2}-\delta m_{L}^{2}\right) \int g^{2}(x): \phi^{2}(x): d x \\
& +\left(E_{K}-E_{L}\right)+\int \tilde{\omega}(p)\left\{\left[\Gamma V_{p r}, b^{*}(p)\right]\left[\Gamma V_{p r}, b(p)\right]\right. \\
& \left.+\left[\Gamma V_{p r}, b^{\prime *}(p)\right]\left[\Gamma V_{p r}, b^{\prime}(p)\right]\right\} d p .
\end{aligned}
$$

The result of normal ordering the fermion operators in the second order term in (7.10) can be represented graphically as

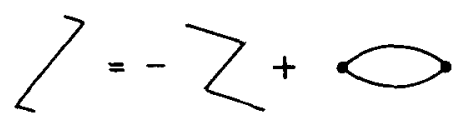

The first term on the right-hand side of $(7.11)$ is the negative of a positive operator; therefore, $(7.10)$ implies

$$
\begin{aligned}
H_{0 F}-N_{\tau F} & +V_{p r}+\frac{1}{2}\left(\delta m_{K}^{2}-\delta m m_{L}^{2}\right) \int g^{2}(x): \phi^{2}(x): d x \\
& +\int d k_{1} d k_{2}: \phi\left(k_{1}\right) q\left(k_{1}, k_{2}\right) \phi\left(-k_{2}\right): \\
& \geqslant-\left(E_{K}-E_{L}\right)-\int d k q(k, k) \mu^{-1}(k),
\end{aligned}
$$

where $q$ denotes the kernel of the last term on the right-hand side of (7.11). The boson fields in this term have also been normal ordered in obtaining (7.12). The right-hand side of $(7.12)$ is equal to [see $(1,5 b)]$

$$
\begin{aligned}
& \left(\frac{\lambda}{4 \pi}\right)^{2} \int_{\left|p_{1}\right|,\left|p_{2}\right| \leqslant K} \frac{\omega_{1} \omega_{2}-p_{1} p_{2}-M^{2}}{\omega_{1} \omega_{2}} r\left(p_{1}, p_{2}\right) \\
& \quad \times\left(\frac{1}{\omega_{1}+\omega_{2}+\mu(k)}-\frac{1}{\widetilde{\omega}_{1}+\widetilde{\omega}_{2}}\right) \mu^{-1}(k)\left|\hat{g}\left(p_{1}+p_{2}+k\right)\right|^{2}(7 \\
& \quad \times d p_{1} d p_{2} d k
\end{aligned}
$$

It can readily be checked that this behaves as - $O(\log K)$. For large $L, c_{1}(\phi)$ can be chosen to be the last two terms on the left-hand side of (7.12), i. e., 


$$
\begin{aligned}
& \left(\frac{\lambda}{4 \pi}\right)^{2} \int d \xi: \phi\left(k_{1}\right) \hat{g}\left(k_{1}-\xi\right)\left(\int_{\substack{p_{1}+p_{2}=\xi \\
\left|p_{1}\right|,\left|p_{2}\right| \leqslant K}} \frac{\omega_{1} \omega_{2}-p_{1} p_{2}-M^{2}}{\omega_{1} \omega_{2}}\right. \\
& \quad \times r\left(p_{1}, p_{2}\right) \frac{1}{\widetilde{\omega}_{1}+\tilde{\omega}_{2}}-\int_{\substack{p_{1}+p_{2}=0 \\
\left|p_{1}\right|,\left|p_{2}\right| \leqslant K}} \frac{\omega_{1} \omega_{2}-p_{1} p_{2}-M^{2}}{\omega_{1} \omega_{2}} r\left(p_{1}, p_{2}\right) \\
& \left.\quad \times \frac{1}{\omega_{1}+\omega_{2}}\right) \hat{g}\left(\xi-k_{2}\right) \phi\left(-k_{2}\right): d k_{1} d k_{2} .
\end{aligned}
$$

(7.8b) is a consequence of an $N_{\tau B}$ estimate (Ref. 8).

Let $q^{\prime}\left(k_{1}, k_{2}\right)$ denote the kernel of $(7.14)$ so that $(7.14)$ equals

$$
\int d k_{1} d k_{2}: \phi\left(k_{1}\right) q^{\prime}\left(k_{1}, k_{2}\right) \phi\left(-k_{2}\right): \text {. }
$$

Then (7.8b) holds if the $L^{2}$ norm of $\mu^{-1}\left(k_{1}\right) q^{\prime}\left(k_{1}, k_{2}\right) \mu^{-1}\left(k_{2}\right)$ can be made arbitrarily small, uniformly in $K$, by choosing $L$ large. The estimates for this are omitted. See (6.17).

Next it is shown that, in $(7.8 \mathrm{c}, \mathrm{d}), c_{2}(\phi)$ can be chosen to be

$$
\begin{aligned}
c_{2}(\phi)= & \frac{1}{2} \int_{\left|p_{1}\right|,\left|p_{2}\right| \leqslant K} r\left(p_{1}, p_{2}\right) W_{s}\left(p_{1}, p_{2}, k_{1}\right) \frac{1}{\omega_{2}^{\tau}} \bar{W}_{s}\left(p_{1}, p_{2}, k_{2}\right) \\
& \times: \phi\left(k_{1}\right) \phi\left(-k_{2}\right): d p_{1} d p_{2} d k
\end{aligned}
$$

For, by an $N_{\tau F}$ estimate to $V_{s r}$, regarding the $\phi$ 's as numerical quantities,

$$
\left\|V_{s r} N_{\tau F}^{-1 / 2}\right\|_{F} \leqslant\left\{2\left[c_{2}(\phi)+O\left(K^{\epsilon}\right)\right]\right\}^{1 / 2} .
$$

The $O\left(K^{\varepsilon}\right)$ arises when the bosons are normal ordered to comply with (7.16). (7.17) implies

$$
V_{s r}^{2} \leqslant 2\left[c_{2}(\phi)+O\left(K^{\epsilon}\right)\right] N_{T F},
$$

and since operator inequalities are preserved in the taking of square roots,

$$
\pm V_{s r} \leqslant \sqrt{2}\left[c_{2}(\phi)+O\left(K^{\epsilon}\right)\right]^{1 / 2} N_{\tau F}^{1 / 2} \leqslant c_{2}(\phi)+O\left(K^{\epsilon}\right)+\frac{1}{2} N_{T F} .
$$

The expression under the square root is equal to $(7.16)$ with the $\phi$ 's not normal ordered. This is a positive operator so the square root is well-defined. (7.18) is equivalent to $(7.8 \mathrm{c})$.

(7. 8d) is proved by an $N_{\tau B}$ estimate. From (7.16), it suffices to show that the $L^{2}$ norm of $(7.19)$ can be made arbitrarily small, uniformly in $K$, by choosing $L$ large:

$$
\begin{aligned}
& \mu\left(k_{1}\right)^{-1} \int_{\left|p_{1}\right|,\left|p_{2}\right| \leqslant K} W_{s}\left(p_{1}, p_{2}, k_{1}\right) \omega^{-\tau}\left(p_{2}\right) \bar{W}_{s}\left(p_{1}, p_{2}, k_{2}\right) r\left(p_{1}, p_{2}\right) \\
& \quad \times d p_{1} d p_{2} \mu\left(k_{2}\right)^{-1}
\end{aligned}
$$

Since this is the kernel of a positive operator on $L^{2}(R)$, the $L^{2}$ norm is less than its trace which is less than

$$
\begin{aligned}
& o(1) \int d \xi|\hat{g}(\xi)|^{2} \int_{p_{1}+p_{2}+k=\xi} \frac{\omega_{1} \omega_{2}+p_{1} p_{2}+M^{2}}{\omega_{1} \omega_{2}} \\
& \times r\left(p_{1}, p_{2}\right) \omega^{-\tau}\left(p_{2}\right) \mu^{-2}(k) .
\end{aligned}
$$

The following estimate applied to $(7.20)$ completes the proof of (7.8d). For $0<\epsilon \leqslant 1$

$\left(\omega_{1} \omega_{2}+p_{1} p_{2}+M^{2}\right) / \omega_{1} \omega_{2} \leqslant O(1) \omega\left(p_{1}+p_{2}\right)^{2 \epsilon} \omega\left(p_{1}-p_{2}\right)^{-\epsilon} .(7.21)$

\section{Generalized Yukawa}

The estimates that substitute for $(7.1 \mathrm{a}, \mathrm{b})$ are: Given $K$ and $\epsilon>0$ there exists $c(\phi)$ such that

$$
\begin{aligned}
& H_{0 F}+V+c(\phi) \geqslant \int g(x): \phi^{2 M}(x): d x-O\left(K^{\epsilon}\right), \\
& H_{0 B}+\int g(x): \phi^{2 M}(x): d x-2 c(\phi) \geqslant-O\left(K^{\varepsilon}\right),
\end{aligned}
$$

where $M>N$ and

$$
\begin{aligned}
V= & \lambda \int: \bar{\psi}_{K}(x) \psi_{K}(x):: \phi^{N}(x): g(x) d x \\
& -\frac{1}{2} \delta m_{K, 0}^{2} \int g^{2}(x): \phi^{2 N}(x): d x \\
& -\frac{1}{2} \delta m_{K, 1}^{2} \int g^{2}(x): \phi^{2 N-2}(x): d x \\
& -\cdots-\frac{1}{2} \delta m_{K, N-1}^{2} \int g^{2}(x): \phi^{2}(x): d x \\
& -E_{K}+\int g(x): \phi^{2 M}(x): d x .
\end{aligned}
$$

$E_{K}$ is not the same as that in (1.5b). The extra counterterms are added to cancel diagrams like

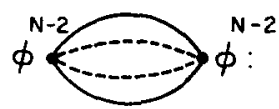

Glimm's dressing transformation is used to bound the pair creation and annihilation part of $V$ by a function $c_{1}(\phi)$ without using a lower momentum cutoff $r\left(p_{1}, p_{2}\right)$. To bound the scattering part, a function $c_{2}(\phi)$ is found so that for $\tau$ close enough to 1

$$
\frac{1}{2} N_{\tau F}+V_{s}+c_{2}(\phi) \geqslant 0,
$$

where

$$
\begin{aligned}
V_{s}= & \int_{\left|p_{1}\right|,\left|p_{2}\right| \leqslant K}\left[b^{*}\left(p_{1}\right) b\left(-p_{2}\right)+b^{* *}\left(p_{1}\right) b^{\prime}\left(-p_{2}\right)\right] W_{s}\left(p_{1}, p_{2}, k\right) \\
& : \phi^{N}(k): d p_{1} d p_{2} d k .
\end{aligned}
$$

: $\phi^{N}(k)$ : denotes the $k$ th Fourier component of : $\phi^{N}(x)$ :. To obtain $c_{2}(\phi)$, begin by estimating, for $\alpha>1 / 2$,

$$
\left\|N_{\tau F}^{-\alpha / 2} V_{s} N_{\tau F}^{-\alpha / 2}\right\|_{F}
$$

Let

$$
y\left(p_{1}, p_{2}\right)=\int W_{s}\left(p_{1}, p_{2}, k\right): \phi^{N}(k): d k .
$$

Then

$$
\begin{aligned}
& N_{\tau F}^{-\alpha / 2} \int_{\substack{p_{1} \geqslant p_{2} \\
\left|p_{1}\right|,\left|p_{2}\right| \leqslant K}} y\left(p_{1}, p_{2}\right) b^{*}\left(p_{1}\right) b\left(-p_{2}\right) d p_{1} d p_{2} N_{\tau F}^{-\alpha / 2} \\
& =O(1) \int_{0}^{\infty} d t t^{-1+\alpha / 2} \exp \left(-t N_{\tau F}\right) \int_{\substack{p_{1} \geqslant p_{2} \\
\left|p_{1}\right|_{,}\left|p_{2}\right| \leqslant K}} \\
& \times y\left(p_{1}, p_{2}\right) b^{*}\left(p_{1}\right) b\left(-p_{2}\right) d p_{1} d p_{2} N_{\tau F}^{-\alpha / 2} \\
& =O(1) \int_{0}^{\infty} d t t^{-1+\alpha / 2} \int_{\substack{p_{1} \geqslant p_{2} \\
\left|p_{1}\right|,\left|p_{2}\right| \leqslant K}}^{\infty} y\left(p_{1}, p_{2}\right) \\
& \times \exp \left[-\left(2 \omega_{1}^{\tau}-\omega_{2}^{\tau}\right) t / 2\right] b^{*}\left(p_{1}\right) \\
& \left.\times \exp [-t / 2) N_{\tau F}\right] b\left(-p_{2}\right) \exp \left[(-t / 2) N_{\tau F}\right] d p_{1} d p_{2} N_{\tau F}^{-\alpha / 2} .
\end{aligned}
$$

Therefore, the \|\|$_{F}$ norm of (7.29) is bounded by 


$$
\begin{aligned}
O(1) \int_{0}^{\infty} d t t^{-1+\alpha / 2} \| \iint_{\substack{p_{1} \geqslant p_{2} \\
\left|p_{1}\right|,\left|p_{2}\right| \leqslant K}} y\left(p_{1}, p_{2}\right) \\
\quad \times \exp \left[-\left(2 \omega_{1}^{\tau}-\omega_{2}^{\tau}\right) t / 2\right] b^{*}\left(p_{1}\right) \\
\left.\quad \times \exp [(-t / 2)] N_{\tau F}\right] b\left(-p_{2}\right) N_{\tau F}^{-1 / 2}\left\|_{F}\right\| N_{\tau F}^{1 / 2-\alpha / 2} \\
\quad \times \exp \left[(-t / 2) N_{\tau F}\right] \|
\end{aligned}
$$

Despite the fact that $\exp \left[(-t / 2) N_{\tau F}\right]$ is sandwiched be-

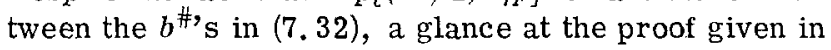
Ref. 8 shows that an $N_{\tau}$ estimate can still be applied so that $(7,32)$ is bounded by

$$
\begin{aligned}
& O(1) \int_{0}^{\infty} d t t^{-1+\alpha / 2} \exp [-O(1) t] t^{-1 / 2+\alpha / 2} \\
& \left.\quad \times\left(\int_{\substack{p_{1} \geqslant p_{2} \\
\left|p_{1}\right|,\left|p_{2}\right| \leqslant K}}\left|y\left(p_{1}, p_{2}\right)\right|^{2} \exp \left[-\left(2 \omega_{1}^{\tau}-\omega_{2}^{\tau}\right) t\right] \omega_{2}^{-\tau} d p_{1} d p_{2}\right)\right)^{1 / 2} \\
& \quad \leqslant O(1)\left[\int\left|y\left(p_{1}, p_{2}\right)\right|^{2} \omega_{1}^{-\epsilon \tau} \omega_{2}^{-\tau} d p_{1} d p_{2}\right]^{1 / 2}
\end{aligned}
$$

provided $\alpha-\frac{3}{2}-\epsilon<1$. It is also necessary that $\epsilon \tau+\tau>1$ so that the boson fields in (7.34) can be normal ordered. Clearly the same estimate can be derived for a term with $p_{1} \leqslant p_{2}$; also antifermions can be included so that

$$
\begin{aligned}
& \left\|N_{\tau F}^{-\alpha / 2} V_{S^{N}} N_{\tau F}^{-\alpha / 2}\right\|_{F} \leqslant O(1)\left[\int\left|y\left(p_{1}, p_{2}\right)\right|^{2}\right. \\
& \left.\times\left(\omega_{1}^{-\epsilon \tau} \omega_{2}^{-\tau}+\omega_{1}^{-\tau} \omega_{2}^{-\epsilon \tau}\right) d p_{1} d p_{2}\right]^{1 / 2} .
\end{aligned}
$$

(7. 35) implies

$$
\pm V_{s} \leqslant O(1)\left[\int\left|y\left(p_{1}, p_{2}\right)\right|^{2}\left(\omega_{1}^{-\epsilon \tau} \omega_{2}^{-\tau}+\omega_{1}^{-\tau} \omega_{2}^{-\epsilon \tau}\right) d p_{1} d p_{2}\right]^{1 / 2} N_{\tau F}^{\alpha}
$$

and therefore

$$
\begin{aligned}
-V_{s} & \leqslant \frac{1}{2} N_{\tau F}+O(1)\left[\int\left|y\left(p_{1}, p_{2}\right)\right|^{2}\right. \\
& \left.\times\left(\omega_{1}^{-\epsilon \tau} \omega_{2}^{-\tau}+\omega_{1}^{-\tau} \omega_{2}^{-\epsilon \tau}\right) d p_{1} d p_{2}\right]^{1 / 2(1-\alpha)},
\end{aligned}
$$

where

$$
\alpha-\frac{3}{2}-\epsilon<1, \quad \epsilon \tau+\tau>1 .
$$

Consequently, (7.25) holds with $c_{2}(\phi)$ chosen to be the last term on the right-hand side of (7.37). Since the dressing transformation only works for $\tau<1,(7.37 \mathrm{~b})$ requires $\alpha>\frac{1}{2}$ 。

To prove (7.22b), one needs, for suitable $\alpha$,

$$
H_{0 B}+\int g(x): \phi^{2 M}(x): d x-2 c_{1}(\phi)-2 c_{2}(\phi) \geqslant-O\left(K^{\varepsilon}\right),
$$

where $c_{1}(\phi)$ is the term arising from the dressing transformation. Instead, it is now proved that $\alpha$ can be chosen so that

$$
\frac{1}{2} H_{0 B}+\frac{1}{2} \int g(x): \phi^{2 M}(x): d x-2 c_{2}(\phi) \geqslant-O\left(K^{\epsilon}\right)
$$

because the estimate with $c_{2}$ replaced by $c_{1}$ in $(7.39)$ can be proved in a similar way to $(7.39) . c_{2}(\phi)$ has the form

$\left[\int Y\left(k_{1}, k_{2}\right): \phi^{N}\left(k_{1}\right):: \phi^{N}\left(-k_{2}\right): d k_{1} d k_{2}\right]^{1 / 2(1-\alpha)}$, where

$$
\begin{aligned}
Y\left(k_{1}, k_{2}\right)= & O(1) \int d \xi \hat{g}\left(k_{1}-\xi\right) \int_{p_{1}+p_{2}=\xi} \frac{1}{\omega_{1}^{\epsilon \tau}} \frac{1}{\omega_{2}^{\tau}}+\frac{1}{\omega_{2}^{\tau}} \frac{1}{\omega_{1}^{\epsilon \tau}} \\
& \times \frac{\omega_{1} \omega_{2}+p_{1} p_{2}+M^{2}}{\omega_{1} \omega_{2}} g\left(\xi-k_{2}\right) .
\end{aligned}
$$

By using the hypothesis $M>N, \alpha$ is picked so that

$$
\alpha>\frac{1}{2}, 1 / 2(1-\alpha)<M / N \text {. }
$$

Let $\gamma=1 / 2(1-\alpha)$. Note that $1<\gamma<M / N$.

By hypercontractivity and the F. K. N. formula (Ref. 7), it is sufficient to prove

$$
\exp \left(-\int_{0}^{1}\left[U(t)-O(1) T^{\gamma}(t)\right] d t\right) \in L^{p}
$$

for $1 \leqslant p<\infty$, where

$$
\begin{aligned}
& U(t)=\int g(x): \phi^{2 \cdot H}(x, t): d x, \\
& T(t)=\int Y\left(k_{1}, k_{2}\right): \phi^{N}\left(k_{1}, t\right): \phi^{N}\left(-k_{2}, t\right): d k_{1} d k_{2} .
\end{aligned}
$$

$\left[\phi(x, t), \phi(k, t)\right.$ refer to Euclidean fields. ] Define $U_{L}(t)$ and $T_{L}(t)$ by introducing a sharp momentum cutoff at $L$ into each field in the spatial momentum. Let

$$
\delta U_{L}(t)=U(t)-U_{L}(t), \quad \delta T_{L}(t)=T(t)-T_{L}(t) .
$$

The following estimate is easy to show, for each $L$ :

$$
\begin{aligned}
\int_{0}^{1} d t\left[U(t)-O(1) T^{\gamma}(t)\right] & \geqslant \int_{0}^{1} d t\left[U_{L}(t)-O(1) T_{L}^{\gamma}(t)\right] \\
& +\int_{0}^{1} d t\left[\delta U_{L}(t)-O(1)\left|\delta T_{L}(t)\right|^{\gamma}\right] .
\end{aligned}
$$

Suppose the estimates

$$
\begin{aligned}
\int_{0}^{1} d t & {\left[U_{L}(t)-O(1) T_{L}^{\gamma}(t)\right] } \\
& \geqslant O(1) \log ^{M} L\left\|\int_{0}^{1} d t\left[\delta U_{L}(t)-O(1)\left|\delta T_{L}(t)\right|^{\gamma}\right]\right\| q \\
& \leqslant O(1)(q \gamma)^{M} L^{-\eta}
\end{aligned}
$$

hold for some $\eta>0$ and all $L$; then Nelson's method (Ref. 9) can be applied to prove (7.42). Therefore, it suffices to prove $(7.47 \mathrm{a}, \mathrm{b})$.

Proof of (7. 47a): In (7.40), $\epsilon \tau+\tau>1$; therefore,

$$
\int_{p_{1}+p_{2}=\xi} \frac{1}{\omega_{1}^{\epsilon \tau}} \frac{1}{\omega_{2}^{\tau}}+\frac{1}{\omega_{2}^{\tau}} \frac{1}{\omega_{1}^{\epsilon \tau}} \leqslant O(1) .
$$

This implies that, considered as operators on $L^{2}(R)$,

$$
Y \leqslant O(\mathbf{1}) \hat{g} * \hat{g}
$$

therefore,

$$
T_{L}(t) \leqslant O(1) \int g^{2}(x)\left(: \phi_{L}^{N}(x, t):\right)^{2} d x .
$$

(7. 50) implies

$\left[U_{L}(t)-O(1) T_{L}^{\gamma}(t)\right] \geqslant U_{L}(t)-O(1)\left[\int g^{2}(x)\left(: \phi_{L}^{N}(x, t):\right)^{2} d x\right]^{\gamma}$.

Since $\gamma=1$ and by hypothesis (1.4) $g \in L^{p}$ for all $p$ $(1 \leqslant p \leqslant \infty)$, the "Hölder" inequality can be applied to (7.51) so that

$$
\begin{gathered}
U_{L}(t)-O(1) T_{L}^{\gamma}(t) \geqslant U_{L}(t)-O(1) \int g(x)\left|: \phi_{L}^{N}(x, t):\right|^{2 \gamma} d x \\
=\int g(x)\left[: \phi_{L}^{2 M}(x, t):-O(1)\left|: \phi_{L}^{N}(x, t):\right|^{2 \gamma}\right] d x .
\end{gathered}
$$

Therefore, (7.47a) is proved if it is shown that

$$
: \phi_{L}^{2 M}(x, t):-O(1)\left[: \phi_{L}^{N}(x, t):: \phi_{L}^{N}(x, t):\right]^{\gamma} \geqslant O(1) \log ^{M H} L .
$$

This follows from Wick's theorem since $\gamma<M / N$.

Proof of $(7.47 \mathrm{~b})$ : This is a consequence of hypercontractivity, e.g. , 


$$
\begin{aligned}
& \left\|\int_{0}^{1} d t\left(\delta U_{L}(t)-O(1)\left|\delta T_{L}(t)\right|^{\gamma}\right)\right\|_{q} \\
& \quad \leqslant \int_{0}^{1} d t\left\|\delta U_{L}(t)\right\|_{\alpha}+O(1) \int_{0}^{1} d t\left\|\delta T_{L}(t)\right\|_{a \gamma}^{\gamma} .
\end{aligned}
$$

Now apply Nelson's best estimate to each term separately on the right-hand side. (For Nelson's best estimate, see Ref. 10).

\section{APPENDIX: UNIFORMITY IN THE EXPECTATION STATE}

It is sufficient to find a uniform lower bound for

$$
-\lim _{T \rightarrow \infty}(1 / T) \ln \left\langle s^{\prime} e^{-T H} s\right\rangle,
$$

where $s$ and $s^{\prime}$ have the form of a product of a Wick monomial in Fermi fields with an $L^{\infty}$ function on $Q$ space depending on time zero fields. Finite sums of quantities of this type are dense, and, by virtue of the $T$ limit, (1.1) evaluated for such a sum is bounded below by the infimum over cross product contributions. The $L^{\infty}$ functions can be majorized by their sup norms during "defermiation." The resulting constant gives no contribution in the $T$ limit. The fermion parts of $s$ and $s^{\prime}$ can be introduced in the calculation by allowing the " $P$ " vertices to contract with the "external" fields in $s$ and $s^{\prime}$. Suppose the latter have been labelled
$1,2, \ldots, p$, then the inclusion of a factor $\left(\Pi_{1} 4^{n(I)}\right)(p !)$ in (4.4) is sufficient to count the extra fermion graphs. A " $P$ " vertex has two legs, each of which can be external or internal, hence the $\Pi_{T} 4^{n(I)}$. In the set of external legs, the first has $p$ choices of external fields with which to contract, the second has $p-1$, etc. ; hence the $p$ !. The $\Pi_{I} 4^{n(I)}$ can be absorbed into the $O(1)^{n(I)}$ in (4.4)。 The $T$ limit annihilates the $p !$; hence a uniform bound holds for (1.1), and the Hamiltonian is bounded below.

*Junior Fellow, University of Michigan Society of Fetlews.

'J. Glimm, Comm. Math. Phys. 5, 343 (1967); 6, 120 (1967).

${ }^{2}$ P. Federbush, J. Math. Phys. 14, 1532 (1973).

${ }^{3} \mathrm{~J}$. Glimm and A. Jaffe, Comm. Math. Phys. 22, 253 (1971).

${ }^{4}$ J. Glimm and A. Jaffe, Fortschr. Phys. 21, 327 (1973).

${ }^{5} \mathrm{D}$. Brydges and P. Federbush, J. Math. Phys. 15, 730 (1974).

${ }^{6} \mathrm{~J}$. Dimock and J.Glimm, "Measures on Schwartz Distribution Space and Applications to $P(\phi)_{2}$ Field Theories," N.Y.U. preprint.

${ }^{7} \mathrm{~F}$. Guerra, L. Rosen, and B. Simon, "The $P(\phi)_{2}$ Euclidean Quantum Field Theory as Classical Statistical Mechanics,"

Ann. Math. (to appear).

${ }^{8} \mathrm{~J}$. Glimm and A. Jaffe, in Les Houches Lecture Notes 1970, edited by C. DeWitt and R. Stora (Gordon and Breach, New York, 1970).

${ }^{9} \mathrm{E}$. Nelson, $A$ Quartic Interaction in Two Dimensions, edited by R. Goodman and I. Segal (M.I. T. Press, Cambridge, 1966).

${ }^{10}$ E. Nelson, Journ. Funct. Anal. 12, 211 (1973). 